\title{
Swabs to genomes: a comprehensive workflow
}

Madison I Dunitz, Jenna M Lang, Guillaume Jospin, Aaron E Darling, Jonathan A Eisen, David A Coil

The sequencing, assembly, and basic analysis of microbial genomes, once a painstaking and expensive undertaking, has become much easier for research labs with access to standard molecular biology and computational tools. However, there are a confusing variety of options available for DNA library preparation and sequencing, and inexperience with bioinformatics can pose a significant barrier to entry for many who may be interested in microbial genomics. The objective of the present study was to design, test, troubleshoot, and publish a simple, comprehensive workflow from the collection of an environmental sample (a swab) to a published microbial genome; empowering even a lab or classroom with limited resources and bioinformatics experience to perform it. 


\section{Swabs to Genomes: A Comprehensive}

\section{Workflow}

4

5

6

16

17

18
Madison I. Dunitz ( $1 *)$

Jenna M. Lang (1*)

Guillaume Jospin (1)

Aaron E. Darling (2)

Jonathan A. Eisen(1\#)

David A. Coil (1)

(1) UC Davis, Genome Center

(2) ithree institute, University of Technology Sydney, Australia

(*) These authors contributed equally to this work.

(\#) Corresponding author: jaeisen@ucdavis.edu 
19

\section{Abstract}

The sequencing, assembly, and basic analysis of microbial genomes, once a painstaking and expensive undertaking, has become much easier for research labs with access to standard molecular biology and computational tools. However, there are a confusing variety of options available for DNA library preparation and sequencing, and inexperience with bioinformatics can pose a significant barrier to entry for many who may be interested in microbial genomics. The objective of the present study was to design, test, troubleshoot, and publish a simple, comprehensive workflow from the collection of an environmental sample (a swab) to a published microbial genome; empowering even a lab or classroom with limited resources and bioinformatics experience to perform it. 


\section{$38 \quad 1$ Introduction}

39 Thanks to decreases in cost and difficulty, sequencing the genome of a

40 microorganism is becoming a relatively common activity in many research and

41 educational institutions. However, such microbial genome sequencing is still far from

42 routine or simple. The objective of this work was to design, test, troubleshoot, and

43 publish a comprehensive workflow for microbial genome sequencing, encompassing

44 everything from culturing new organisms to depositing sequence data; enabling even

45 a lab with limited resources and bioinformatics experience to perform it.

In late 2011, our lab began a project with the goal of having undergraduate students generate genome sequences for microorganisms isolated from the "built environment". The project focused on the built environment because it was part of the larger "microBEnet" (microbiology of the built environment network,

50 www.microbe. net) effort. This project serves many purposes, including (1)

51 engaging undergraduates in research on microbiology of the built environment, (2)

52 generating "reference genomes" for microbes that are found in the built environment,

53 and (3) providing a resource for educational activities on the microbiology of the built

54 environment. As part of this project, undergraduate students isolated and classified

55 microbes, sequenced and assembled their genomes, submitted the genome sequences

56 to databases housed by The National Center for Biotechnology Information (NCBI),

57 and published the genomes [31][7][24][17][12][27]. Despite the reduced cost of

58 genome sequencing and the availability of diverse tools making many of the steps

59 easier, (e.g., kits for library prep, cost-effective sequencing, bioinformatics pipelines),

60 there were still a significant number of stumbling blocks. Moreover, some portions of

61 the project involve choosing between a wide variety of options (e.g., choice of 
assembly program) which can create a barrier for a lab without a bioinformatician.

Each option comes with its own advantages and disadvantages in terms of complexity, expense, computing power, time, and experience required. In this workflow, we describe an approach to genome sequencing that allows a researcher to go from a swab to a published paper (Figure 1). We used this workflow to process a novel Tatumella sp. isolate and publish the genome [19]. The data from every step of the workflow, using this Tatumella isolate, is available on Figshare [13]

The sequencing and de novo assembly of genomes has yielded enormous scientific insight revolutionizing a wide range of fields, from epidemiology to ecology. Our hope is that this workflow will help make this revolution more accessible to all scientists, as well as present educational opportunities for undergraduate researchers and classes.

There are several excellent resources that focus on smaller portions of this entire workflow. Examples include the Computational Genomics Pipeline [29] and a "Beginner's guide to comparative bacterial genome analysis" [23]. Clarke et. al., 2014 describes a similar pipeline focused on human mitochondrial genomes [10].

\section{Background}

\subsection{Background: bioinformatics}

\subsubsection{Command Line/Terminal Tutorial}

This workflow is written assuming that the user is using a computer running Mac OS $\mathrm{X}$ or Linux. It is also possible to carry out many of the computational parts of this 
workflow in a Windows environment but getting these steps to work in Windows is outside the scope of this project.

Some parts of this workflow require the user to provide text instructions for software programs by using a command line interface. While potentially intimidating to computer novices, the use of command line interfaces is sometimes necessary $(e . g$, some programs do not have graphical interfaces) and is also sometimes much more efficient. To access the command line on a Mac open the Terminal program (the default location for this program is in the "Utilities" folder under "Applications").

When this application is launched, a new window will appear. This is known as a "terminal" or a "terminal window". In the terminal window, you can interact with your computer without using a mouse. Many popular programs have a GUI (Graphical User Interface) but some programs used in this workflow will not. So, instead of double-clicking to make a program run, you will type a command in the terminal window. Throughout this tutorial, we will instruct you to type commands, but copying and pasting them (when possible) will reduce the occurrence of typos. We will walk you through how to run all of the programs required for this workflow, but you must first acquire a basic familiarity with how to interact with your computer through the terminal window. Below is a list of commands that will be required to use this workflow. There are many tutorials available to help you get started.

For more information on operating in the terminal, check out this informative video: https: / /www. youtube. com/watch?v=zRZT4nQP 3sE

And this interactive tutorial:

http://www.ee.surrey.ac.uk/Teaching/Unix/ 


\subsubsection{Summary of Unix/Linux commands and terms}

107 \$ Is lists files and directories (folders). If left as just "ls" this command will list the

108 files and directories in your current location. If a "path" is added afterwards (e.g., ls

109 /usr) this command will list the files and directories in that location.

110 \$ cd use to change directories

111 \$ cd .. use to move up one directory

112 \$ cd directory_name use to move to that directory

113 \$ cd $\sim$ use to move to the home directory of the current user

114 \$ grep "some pattern" file_name displays lines that match the pattern

115 (contained within the quotes) for which you are searching. If a line contains the same

116 character multiple times it will only be displayed once.

117 \$ grep -c "what you want to count" file_name counts the number of lines

118 containing a specific character or sequence of characters

119 \$ less file_name view a file, type $\mathrm{q}$ to exit

$120 \quad$ A few quick definitions:

121 command line - the command line is where you type commands in a terminal

122 window

123 script - a computer program. Usually computer programs are called scripts when

124 they perform relatively simple functions that are limited in scope. Scripts are typically

125 only run from the command line

126 directory - a folder 
compile - turning a human-readable file into a computer-executable program

128

129

130

131

132

133

134

135

136

137

138

139

140

141

142

143

144

145

146

147 (methods for carrying out lab experiments without contamination from living

148 microorganisms). The starting point is the collection of microbes from a surface with

149 a swab. We will cover the steps necessary to take a sample through plating, dilution

150 streaking, overnight growth, creating a glycerol stock, 16S rDNA PCR, and 
151 preparation for Sanger sequencing to determine the identity of your bacterial or

152 archaeal isolate.

153 Throughout the "Isolation" section we refer frequently to "media" and "culture

154 media". This is in reference to the type of substrate (sometimes liquid, sometimes a

155 gel-like material such as agar) used to grow microbes in the lab. The choice of media

156 will depend on the goals of the particular project. Some factors to consider when

157 selecting media and conditions for growth include:

$158 \quad$ 1. What type of organism do you want to isolate?

1592 . Are there types of organisms (e.g., pathogens) that you would prefer not to

160 isolate? For example, swabbing people and growing samples on blood agar at

$161 \quad 37^{\circ} \mathrm{C}$ can preferentially isolate human pathogens.

162 3. How much time is available for growth and isolation?

163

164

- growth rates differ both between organisms (e.g., species 1 versus

165 species 2) and also in different conditions for the same organisms (e.g.,

$166 \quad$ species 1 at $20^{\circ} \mathrm{C}$ vs. $37^{\circ} \mathrm{C}$ )

167 - for many microbes there is an "optimal growth temperature" (OGT -

168 the temperature at which it grows best) but the OGT varies between

169 species

170 - you will be able to isolate a greater diversity of organisms if you allow

171 a long time for slow-growing organisms to grow

172 4. What types of equipment are available to you? 
- if an organism grows most happily at $37^{\circ} \mathrm{C}$, then you will need to have an incubator and shaker available at that temperature. preparation and agar plates, we recommend the following resource:

\subsection{Background: phylogeny and systematics.}

181 In order to identify to which organism a $16 \mathrm{~S}$ rDNA sequence belongs, as well as to

182 provide an evolutionary context for your organism of interest, we recommend

183 inferring a phylogenetic tree (see Section 7). Building such a phylogenetic tree is

184 (relatively speaking) the easy part. Intelligent interpretation of the tree will require an

185 investment of time, similar to the investment required to learn the basics of UNIX.

186 Fortunately, there are a number of resources available for this purpose. We

187 recommend this online tutorial

188 (http://evolution.berkeley.edu/evolibrary/article/phyloge

189 netics_02) or [4] Here we provide a brief introduction to phylogenetic trees.

190 A phylogenetic tree is a diagram representing a model of evolutionary

191 relationships. Phylogenetic trees have three main components: taxa, branches, and

192 nodes (Figure 2). These are defined below:

193 - Taxon. An individual or grouping of individuals. This could be individual

194 sequences, species, families, phyla, etc. For phylogenetic analyses, the taxa 
that are drawn at the tips of branches are sometimes referred to as "leaves" on the tree.

- Branch. A representation of the evolution of a taxon over time (sometimes also known as an evolutionary lineage). There are three main types of branches in a tree. Terminal branches are those that lead to the tips or leaves in the tree. Internal branches connect branches to each other. And the root branch, also known as the root of the tree, is the branch that leads from the base of the tree to the first node in the tree.

- Node. These are the points where individual branches end. In the internal parts of a phylogenetic tree, single branches can "split" producing multiple descendant branches. The point at which the branches split is known as an internal node. If a branch ends at a taxon, the end point is known as a "terminal node".

- Clade. A group of organisms consisting of a single node and all the descendants of that node in a tree and nothing else.

212 - Bootstrapping. A statistical method used to measure how well a node is

213 supported by all the data being used.

214 - Ingroup. The group of taxa being studied.

- Outgroup. A taxon that separated in an evolutionary tree prior to the existence of the most recent common ancestor of the ingroup. 


\section{Isolation}

218 This section will take you through the basics of isolating, culturing, and storing your

219 organism.

\section{$220 \quad 3.1$ Swab}

221 Using a sterile cotton swab (for example the "Sterile Cotton Tipped Applicators"

222 from Puritan), wipe (i.e., "swab") the area you intend to sample for 10 to 15 seconds,

223 as if you were trying to clean the area. Try to rotate the swab to ensure that all sides

224 touch the surface.

$225 \quad 3.2 \quad$ Plate

226 Gently (so as not to break the agar surface) rub, i.e., "streak" the swab across the

227 entire surface of an agar plate. Be sure to rotate the swab as you are doing so to

228 ensure that all sides of the swab make contact with the plate. Incubate the plate at the

229 desired temperature (in our case, usually $37^{\circ} \mathrm{C}$ or room temperature) until colonies

230 appear.

$231 \quad 3.3$ Dilution Streak (streaking for individual

232 colonies) $\mathbf{x} 2$

233 After incubation, choose desired colonies (we typically attempt to maximize the

234 diversity of colony morphologies) and dilution streak them onto individual plates. 
235 Dilution streaking involves spreading out a chosen colony such that new single

236 colonies grow on a new plate (details can be found online).

237 After growth to visible colonies, repeat the dilution streaking to help ensure purity

238 of the culture. Some organisms will only grow in tight association with others, and a

239 mixed culture will prove difficult to classify and assemble.

\section{$240 \quad 3.4$ Liquid Culture}

241 After the second dilution streaking, a liquid culture is needed both for long-term

242 storage and for DNA extraction. Transfer a single colony from each dilution streak

243 plate into $5 \mathrm{mls}$ of culture media and grow for 1-3 days until cloudy. Once the liquid

244 culture is ready, prepare a $10 \%$ final concentration glycerol stock for long-term

245 storage at $-80^{\circ} \mathrm{C}$ from $1-2 \mathrm{ml}$ of the sample.

\section{16S rDNA Sanger Sequencing}

247 Following liquid culturing, the organisms need to be identified, or classified. This is

248 accomplished by determining and then analyzing the DNA sequence of the 16S rRNA

249 gene. In this section, we describe how the sequence of this gene is determined and

250 readied for analysis. The general outline is as follows: DNA extraction, polymerase

251 chain reaction (PCR) amplification of the 16S rRNA gene, and sequencing of the

252 resulting PCR product using Sanger sequencing [38]. There are multiple approaches

253 one can take to these steps. For example, the PCR requires DNA from the organism

254 of interest. That DNA can come directly from a liquid culture of the organism (when

255 this is used for PCR this is known as colony PCR). Alternatively, one can take a 
256 liquid culture and then isolate the DNA from that culture and use the purified DNA as

257 input material for the PCR. This adds an extra step to the process - a step known as

258 DNA extraction (see below.) Colony PCR significantly decreases the amount of work

259 needed for preparation, but it can yield poorer results, both in terms of PCR success

260 and resultant sequence quality. However, we recommend colony PCR when screening

261 a large number of samples. DNA extraction can then be used for any recalcitrant

262 samples. DNA extraction is significantly more work, but it often generates better

263 Sanger sequences allowing for more accurate identification.

\section{$264 \quad$ 4.1 DNA Extraction}

265 There are a number of different options for DNA extraction, and which one should be

266 used depends on many factors including available equipment, experience, and cost. A

267 standard approach in microbiology involves the use of a phenol and chloroform

268 extraction followed by ethanol precipitation, and any number of protocols for this

269 approach can be found in books, articles and on the internet. A common alternative

270 approach is to use a commercially available kit - there are many advantages to such

271 kits - notably ease and lack of toxic chemicals. A disadvantage of kits is that they

272 typically are more expensive per sample than other approaches (especially if one is

273 only doing a few samples, since most kits include materials for a minimum of 50

274 samples). For most projects, we use kits - typically the Promega-Wizard Genomic

275 DNA Purification Kit.

276 Follow the protocol or kit instructions provided by the manufacturer and then

277 proceed to "PCR" below. 


\section{$278 \quad 4.2$ Colony PCR (if not extracting DNA)}

279 Centrifuge $1 \mathrm{ml}$ of the overnight culture until the cells form a pellet at the bottom of

280 the tube (about 5 minutes at 10,000 g), pour off the liquid on top (the supernatant) and

281 resuspend the pellet in $100 \mu \mathrm{l}$ of sterile DNAase-free water. Incubate the samples at

$282100^{\circ} \mathrm{C}$ for 10 minutes to help lyse the cells. Use the resulting solution as the template

283 in the PCR below.

\section{$284 \quad$ 4.3 PCR}

285 This reaction uses the 27F (AGAGTTTGATCMTGGCTCAG) and 1391R

286 (GACGGGCGGTGTGTRCA) primers which amplify a near full-length bacterial

287 (and many archaeal) 16S rRNA gene. Our lab uses standard PCR reagents (Qiagen or

288 Kappa), with an annealing temperature of $54^{\circ} \mathrm{C}$ and an extension at $72^{\circ} \mathrm{C}$ of 90

289 seconds. Do not forget to include positive (any sample containing bacterial genomic

290 DNA that you have successfully amplified before) and negative (e.g., replace DNA

291 with water) controls. The full program we use is:

$292 \quad 1: 95^{\circ} \mathrm{C}$ for $2: 00$

$293 \quad 2: 95^{\circ} \mathrm{C}$ for $0: 15$

$294 \quad 3: 54^{\circ} \mathrm{C}$ for $0: 30$

$295 \quad 4: 72^{\circ} \mathrm{C}$ for $1: 30$

296 5: Go to 2 (40 times)

$297 \quad 6: 72^{\circ} \mathrm{C}$ for $3: 00$ 
313 not reveal the presence of a Sequencing Facility near you, most sequencing centers

314 will allow you to ship samples to them for sequencing. Another possibility is

315 Science Exchange which is an online clearinghouse for lab services.

7: $4^{\circ} \mathrm{C}$ forever

After PCR is completed, confirm the PCR worked by agarose gel electrophoresis, all controls behaved as expected (i.e., band in the positive control and no band in the negative control), and that you have DNA fragments of the correct size ( 1350bp).

\subsection{Submit Samples for Sequencing}

Very few single-researcher labs currently have the capacity to do Sanger sequencing. However, there are a number of DNA sequencing facilities (commercial and academic) that provide Sanger sequencing services for researchers. They will handle as little as a single sample, or will allow you to submit an unlimited number of samples, arrayed in 96-well plates. You will typically provide both your PCR product as well as primers for sequencing (the same primers used for PCR are usually used for sequencing). To get the most data, do not forget to request both forward (e.g., using primer 27F) and reverse (e.g., using primer 1391R) reactions for each sample. Each facility will have its own guidelines concerning DNA and primer concentration. Our lab uses the UC Davis Sequencing Facility. If an internet search does

\section{Sanger Sequence Processing}

317 The end product of Sanger sequencing is the production of sequences (reads) for each

318 sample submitted. Upon receiving Sanger reads from a sequencing facility, typically 
319 as .abi files via email, it is necessary to do some pre-processing before they can be

320 analyzed. These steps include quality trimming the reads, reverse complementing the

321 reverse sequence, aligning the reads, generating a consensus sequence, and

322 converting to FASTA format. There are very limited options for free software that

323 allow the user to perform these steps.

324 In this workflow we recommend using an automated pipeline available at the

325 Ribosomal Database Project [14] if working with a large number of sequences. This

326 pipeline only provides a rough view, since it doesn't orient or align the reads, it

327 simply quality trims them and outputs the data in a format that can be fed directly to

328 the BLAST program at NCBI [2]. This will at least give an idea of which genera, and

329 sometimes which species, to which each sequence can be classified. We then

330 recommend processing samples of interest using SeqTrace [42] which allows the user

331 to see the traces (graphical representation of reads), process the sequences manually,

332 and a get a longer, more accurate sequence for analysis.

333 We have also created a script that will perform the same steps as SeqTrace

334 automatically, but does not allow you to adjust any of the parameters. The choice of

335 our script (easy, little control) versus SeqTrace (more complex, more control) will

336 depend on the user and the project.

\section{$337 \quad 5.1 \quad$ RDP Sanger pipeline}

338 (recommended as a starting place, or when working with many sequences)

339 The RDP Sanger analysis pipeline can be found here

340 https://rdp. cme.msu.edu/login/pipeline/libsummary. 
This pipeline allows you to upload one zipped folder containing multiple abi traces. It cleans and processes the sequences and generates a FASTA file of the processed sequences; which can then be uploaded to BLAST and analyzed. This allows you to quickly screen your samples before running the files through the more time consuming SeqTrace analysis which will reverse complement and align the reads to generate a consensus sequence.

After signing in to RDP, you will be on the "Library Run Summary" page. Click on the "Create New Run" tab near the top of the page. Select the appropriate 16S rRNA gene (Archaea or Bacteria depending on your sample) name your library and choose a library name abbreviation and select any vector (this pipeline assumes cloned PCR fragments but will work fine regardless of what you select here). Select the "Upload the data without well mapping" button at the bottom of the page. You will now be directed to the "Data Loader" page, choose a zipped folder containing the .abi traces you wish to analyze and click "Load Data". To create the folder, put all of the .abi traces you are working with into a folder, right click on the folder and select Compress "folder name". If you downloaded the files as a group from your sequencing facility, they may already be in a zipped folder.

When the pipeline is finished, you will be directed to click a link that will open a new window containing the library run stats. Select the "Download Raw Sequence" button. Navigate to

http://blast.ncbi.nlm.nih.gov/Blast. cgi ?PROGRAM=blastn $\backslash \& P$ AGE_TYPE=BlastSearch $\backslash \& L I N K \_L O C=$ blasthome and select the "Choose File" button underneath the area for the FASTA sequence. Select the file you just downloaded from the library run stats page. We recommend checking the box to exclude Uncultured/environmental sample sequences then click "BLAST". If you are 
367 the BLAST search is complete, you can cycle through the results using the pull down 368 menu to the right of the "Results for:" heading.

\subsection{SeqTrace}

We recommend using SeqTrace first if only working with a couple of sequences.

371 When working with a large batch it might be easier to do a preliminary screening of

372 the sequences using the RDP Sanger pipeline above and only using SeqTrace for

373 sequences of interest.

$374 \quad$ Download the program from

https://code.google.com/p/seqtrace/downloads/list

$376 \quad$ Installation directions

https://code.google.com/p/seqtrace/wiki/Installation

Installing and running SeqTrace on a PC is simple; installing it on a Mac requires

379 a few more steps than for a PC. The installation guide offers two options for installing

380 SeqTrace on a Mac; we recommend running SeqTrace with native GTK+.

381 To install SeqTrace on a Mac, you will need to download the PyGTK package 382 from OSX.

383 http://sourceforge.net/projects/macpkg/files/PyGTK/2.24.0

384 /PyGTK.pkg/download

385 Currently, SeqTrace depends on Python version 2.x. Confirm that you have

386 Python version 2.x. You can do this by typing: 

407 ts

python --version

You should see something that looks like "Python 2.6.9" If you see Python 3.x, seek outside help to run an earlier version.

$$
\text { http: //www.python.org/downloads/ }
$$

After downloading and unpacking the program, SeqTrace is ready for use.

SeqTrace must be launched from a Terminal window. For a refresher or introduction to the Terminal, see Section 2.1. Move SeqTrace to your Applications folder.

Open a Terminal window and copy/paste or type:

\section{/Applications/seqtrace-0.9.0/seqtrace.py}

This syntax will only work if the SeqTrace folder's name is seqtrace-0.9.0, if you saved it under a different name you will need to replace seqtrace-0.9.0 with the name of that folder.

This will launch SeqTrace from the terminal in a Python shell; you will need to keep the terminal window open while you are using the program.

$$
\text { SeqTrace provides excellent instructions for using the program at }
$$

https://code.google.com/p/seqtrace/wiki/WorkingWithProjec

\section{$408 \quad$ 5.2.1 Edit and Create a Consensus Sequence with SeqTrace}

409 For this workflow we have found that the following is the simplest way to edit and

410 create a consensus sequence from forward and reverse reads in SeqTrace. 
412 1. Create a new project (File $>$ New Project). Add your forward and reverse

413 primer sequences here; we used 27F (AGAGTTTGATCMTGGCTCAG) and

414 1391R (GACGGGCGGTGTGTRCA) and click “OK”.

415 2. To add files, go to "Traces" and click on "Add trace files", then select the 416 reads (.abi files) you want to work with.

417 3. The program is able to recognize forward and reverse reads from information 418 in the file name if they are properly formatted.

- Go to "Traces" and click on "Find" and mark forward/reverse. The default setting looks for_F for forward and_R for reverse. This can be edited in the Project settings (you can pull it up by clicking on the picture of the tool at the top of the page) and changing the search strings under trace settings. For an example, see Figure 3.

- If the program is able to recognize the forward/reverse reads it will place an orange left pointing arrow in front of reverse reads and a blue right pointing arrow in front of forward reads. This step is not necessary to get a consensus sequence, it just makes organizing the reads easier.

1. Pull up the "Project Settings" by clicking on the picture of tool at the top of the page. Click on the "Sequence Processing" tab and under "Sequence trimming," unclick the Automatically trim sequence ends button. You should also decrease the Min. confidence score under "Consensus" settings. The 
default option is 30 , which represents $99.9 \%$ accuracy. For many reads this will be too stringent and will not allow you to get enough overlap to create a consensus sequence. A minimum confidence score between 15 and 25 is normally okay but tuning may be required depending on your read quality. For an example, see Figure 4.

2. Group your forward and reverse reads by highlighting both of them and

4. To view your consensus sequence, click on the read pair group and then click

3. Under "Sequences" go to "Generate Finished Sequences" and click on "for all trace files". (you will need to redo this every time you change the project settings). on the magnifying glass at the top of the page. You should see something like Figure 5.

5. The "Trace View" shows the quality scores, the chromatogram (trace) display, and the raw base calls from both the forward and reverse reads, as well as the consensus sequence. The consensus sequence is the middle list of nucleotides. If the program is giving you a string of Ns where your forward and reverse reads do not overlap, you need to decrease the Min. confidence score.

6. To export the consensus from the trace view, go to "Sequence", hover on "Export Sequences", and select "Export Sequences from Selected Trace Files". This will create a file containing the consensus sequence, which can then be used for analysis such as searching for closely related sequences using the BLAST program [2] which can be used to identify the organism. 
$457 \quad 5.3$ Custom Script to Create a Consensus

458 Sequence (merge_sanger_16s.pl)

459 This custom script is for users who prefer to quickly trim and align their sequences. It

460 is to be used in place of SeqTrace, with or without having pre-screened samples using

461 the RDP Sanger pipeline described above.

$462 \quad$ 5.3.1 Download/Install

463

464 1. Create a new folder called "Sanger_seq" on your Desktop

465 2. Download the zip file, containing three scripts (merge_sanger_16s.pl,

$466 \quad$ cleanup.pl and subsample_reads.pl) from [30]

467 3. Open the zip file and move the merge_sanger_16s.pl file to the new

468 "Sanger_seq" folder

$469 \quad$ 5.3.2 MUSCLE

470 In order to run this script you will need to download MUSCLE [22] from here:

471 http://www.drive5.com/muscle/downloads.htm. Uncompress and

472 open the MUSCLE directory, and record the full path and name of the executable file

473 (e.g. muscle3.8.31_i86darwin64) for later use. 
474 475 476

492

493

494

495

496

497

498

\subsubsection{Convert Files from .abi to .fastq}

To run the merge_sanger_16s.pl script, you will first need to convert your read files from .abi to .fastq

$$
\text { This can be done at }
$$

http://sequenceconversion.bugaco.com/converter/biology/se quences/

Use the drop down menus to set it to convert .abi files to .fastq. Upload a file and convert it. The converted file will save to your downloads folder under the name "sample.fastq". If you are working with a lot of reads, we recommend immediately renaming the files to match the original abi file name to avoid confusion.

\subsubsection{Edit and Create a Consensus Sequence}

Once all of your files are in .fastq format, move all of them to the "Sanger_seq' folder in which you saved the merge_sanger_16s.pl script. Use the terminal to navigate to within this folder by typing:

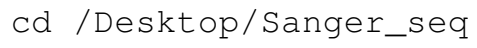

Then, to run the script, type:

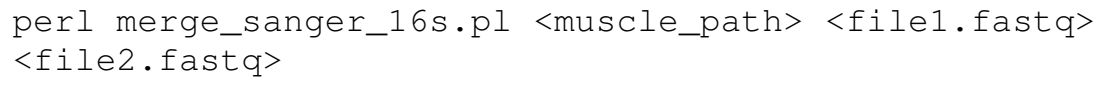


1. "Found $\mathrm{N}$ conflicting case(s) during merging of $\mathrm{X}$ residues"

2. "Not enough data to overlap confidently"

In the first case, the merging happened, however there may be some conflicting

503 bases. The fewer the better. It can be an indication of how confident the user should

504 be with the results. Since this is a very crude method, it should be noted that there is

505 no complex algorithm behind the merge. There is a simple comparison for which we

506 keep the base that had the highest quality score.

507 In the second outcome, the sequences were trimmed too much when doing the

508 quality-trimming. The length of both sequences end to end was smaller than the

509 fragment length that we are looking for. This is an indication of poor quality sequence

510 and most users should not proceed (others can lower the quality threshold set by the

511 script).

512 The newly merged file will be saved as file1_merged.fasta and can be uploaded to

513 BLAST for identification (see Section 6.1).

\section{Organism identification using 16S rRNA gene}

\section{Sequence}

516 It is necessary to screen the $16 \mathrm{~S}$ rDNA Sanger sequencing results for possible

517 genome sequencing candidates. We recommend starting with BLAST results, then

518 continuing onto the Genomes Online Database (GOLD). This is a large database

519 containing most sequenced genomes and many ongoing sequencing projects. 
520 Sometimes the use of GOLD and an internet search will be sufficient to obtain

521 information about the organism you have isolated. In many cases, it will be useful to

522 build a phylogenetic tree to aid in identification, as the BLAST search results may not

523 be sufficiently informative.

\section{$524 \quad$ 6.1 BLAST 16S rDNA sequence}

Begin by navigating to the Standard Nucleotide BLAST at NCBI:

http://blast.ncbi.nlm.nih.gov/Blast.cgi ?PROGRAM=blastn\\&P AGE_TYPE=Blast Search $\backslash \& L I N K \_L O C=b l$ ast home

Paste in your Sanger consensus sequence. We recommend checking the box to exclude Uncultured/environmental sample sequences, since these will not be informative for identification. Be sure the nucleotide collection (nr/nt) is selected under database and click the "BLAST" button (Figure 6).

\subsection{Interpreting the results}

Depending on the quality of the Sanger sequencing and the particular microbe sequenced, the BLAST search results can range from definitive to relatively uninformative. Examples of both are discussed below.

1. In some cases, it is not necessary to build a phylogenetic tree for further identification. If all of the top hits are the same species (or end in sp.), have $e$-values of 0.0 , good query coverage, and $99 \%$ to $100 \%$ identity, you can proceed to "Using GOLD". 
2. In other cases, the results are more ambiguous. The results may show more than $99 \%$ identity to multiple species, or even to multiple genera. In this case, refer to Section 7 "Building a 16S rDNA Tree", before using GOLD.

3. Another possibility is that you will get significantly less than $99 \%$ identity to any sequences in the NCBI database. One explanation for this is that your sequence is of poor quality. This might require more stringent trimming using SeqTrace or even resequencing if the quality is poor enough to make assigning taxonomy difficult. Another possibility is that you have isolated something that is not very closely related to anything in the NCBI database. In the latter case, we would recommend first re-doing the BLAST search, but unchecking "Uncultured/environmental sample" to see if the sequence matches others that have been found, but are not associated with a cultured organism. In either case, we would recommend re-sequencing for confirmation and then refer to Section 7 "Building a $16 \mathrm{~S}$ rDNA Tree" to examine the phylogenetic context of the novel sequence.

\subsection{Using GOLD (the Genomes Online Database)}

Go to: http://genomesonline.org/cgi-bin/GOLD/index.cgi

$$
\text { Under the Search tab, click the "Quick Search" option and you should be taken to }
$$
a page that looks like the screen shot displayed in Figure 7.

Fill out the Biosample name section, with information about your microbe from BLAST and click "Sequencing Project Search". We usually search for only the genus to get a sense for how well that genus is represented in the database and which 
563 species are present. Figure 8 shows an example screen shot of the results for

564 "Brachybacterium." The third column (Project Status) lists the current status of the 565 project (complete, permanent draft, incomplete, targeted). While some "incomplete"

566 and "targeted" projects will be completed, many will not, so we tend to ignore these 567 categories.

568 If you have relatively ambiguous identification results (e.g. you think you have 569 some sort of Brachybacterium but aren't sure which species,) it could be worthwhile 570 to perform an alignment of your $16 \mathrm{~S}$ rDNA sequence with those from genomes 571 already in Genbank or to build a phylogenetic tree as in Section 7.

\section{$572 \quad 6.4$ Compare two 16S rDNA sequences}

573 First locate the 16S rRNA gene sequences of the genome you'd like to compare to, by 574 searching the NCBI Nucleotide database using the name of your species and " $16 \mathrm{~S}$ 575 ribosomal RNA”.

576 http://www.ncbi.nlm.nih.gov/nuccore/

577 Click on the sequence of interest, then click on the "FASTA" link to get the 578 sequence in FASTA format. Now navigate to the "Align Sequences Nucleotide 579 BLAST" page:

580 http://blast.ncbi.nlm.nih.gov/Blast.cgi?PAGE_TYPE=BlastSe

581 arch \\&BLAST_SPEC=blast2seq \\&LINK_LOC=align2seq

582 Paste in the two 16S rDNA sequences and click on the "BLAST" button. Unless

583 both your sequence and the sequence to which you are comparing were amplified 
584 with the same primers, the query coverage will not be $100 \%$ (Figure 9). A low

585 identity can be the result of poor sequence quality or taxonomic distance.

$586 \quad$ 6.5 Choosing an organism to sequence

587 A choice of whether to sequence an organism based on these results depends on the

588 project goal. How closely your isolate is related to an organism with a sequenced

589 genome might be completely irrelevant if you are interested in sequencing your

590 isolate per se, perhaps because of where you found it or because of some interesting

591 phenotype. If your goal is to increase the phylogenetic diversity of available genome

592 sequences, then sequencing the 200th E. coli genome is not the ideal approach to

593 achieve that goal. At the other extreme, if you have isolated an organism that is only

$59490 \%$ identical to anything with a currently available genome sequence, or that appears

595 to be alone on a long branch on your phylogenetic tree, then you have a good

596 candidate to achieve your goal. Of course $90 \%$ is arbitrary, as is a "long" branch, but

597 the current standard is to use a 97\% 16S rRNA gene sequence identity as a proxy for

598 species delimitation in bacteria. This is yet another arbitrary cutoff, and is frequently

599 debated in the field [8][18][26][41]. Finally, you might be interested in increasing the

600 genome sequences available for a particular lineage, for example, to provide

601 additional data for a comparative genomics project. In that case, and in many others,

602 the ideal number of close relatives and the definition of "close," will be unique to

603 each project. 


\section{$604 \quad 7 \quad$ Building a 16S rDNA Tree}

605 Our preferred approach to classifying microbial species is to place the unknown

606 organism in the context of a phylogenetic tree using its 16S rRNA gene sequence.

607 Building a phylogenetic tree from a 16S rRNA sequence is fairly straightforward, but

608 the interpretation of the tree can be a bit complex. Here, we attempt to guide you

609 through both. However, some complicated cases will require consultation with an

610 expert in the field of phylogenetics or systematics.

611 The outline of the workflow is to use the Ribosomal Database Project (RDP) to

612 generate an alignment of the sequence with close relatives and an outgroup. This is

613 followed by cleanup of the RDP headers, tree-building with FastTree [36], and

614 viewing/interpretation of the tree using Dendroscope [28].

\section{$615 \quad 7.1$ Obtain an RDP alignment}

616 The goal of this section is to obtain an alignment of 16S rRNA gene sequences from

617 RDP that can be used to build a tree. This procedure has the added benefit of

618 providing an independent verification of the taxonomic assignment of your sequence

619 based on the BLAST results.

620

621 1. Go to http://rdp.cme.msu.edu

622 2. Create an account

623 3. Click on "my RDP/login" 
624 4. Upload the fasta file containing your $16 \mathrm{~S}$ rDNA sequence

625 5. Assign it a group name (this is what the program will label your sequence/organism). Choose this carefully since that will be the name on the

627 final tree.

628 6. Click the " + " next to the sequence to add it to your cart

629 7. Click on "CLASSIFIER" at the top of the page

630 8. Click on "Do Classification With Selected Sequences" button. This will show 631 you a hierarchical view of the classification of your sequence (from Phylum to 632 Genus.) You will use this information to navigate to other sequences that you 633 want to include in the alignment that you will use to build your phylogenetic 634 tree.

635 9. Click on "BROWSERS". We recommend opening "BROWSERS" in a new 636 tab so that you can keep the hierarchy information handy.

637 10. Click on "Isolates" to select only isolates for further analysis. Then click "Browse" (Figure 10).

11. Click on the + sign next to "Archaea outgroup." This will add an Archaeal 640 sequence to your cart, which will be used to root your phylogenetic tree. Even 641 better would be to chose an outgroup within the same bacterial phyla that you 642 know to be outside of the clade you are examining. If in doubt, just use the 643 Archaeal one.

644 12. If using the example sequence provided, click on "Proteobacteria", then

645 "Gammaproteobacteria", then "Enterobacteriales", then 
"Enterobacteriaceae". This will take you to the Genus Tatumella, which currently has over 69 entries in it. If the genus you are working with has too many sequences to analyze easily (for example, Bacillus currently has $>26,000)$, one way to reduce this number is to exclude the uncultured taxa in the database. To do this, scroll down to the "Data Set Options" and click on the "Isolates" button. Click "Refresh" and you will see that there are fewer sequences in the Genus. To reduce this number further, click on the "Type" Strain button (though if you do this you'll have to build a tree later for species identification since each species will only be represented once in the tree). As a worst-case scenario, you will need to manually select a subset of organisms to include in your alignment.

13. Click on the + sign next to genus Tatumella to add all of those sequences to your cart.

14. Click on "Sequence Cart" and confirm that your uploaded sequence, the outgroup sequence, and all of the other sequences you'd like to include in your tree are displayed.

15. Click on "download," leave the download options as the defaults (fasta, aligned, uncorrected), and then click on the appropriate download button. Save the file and then rename it to something informative.

\subsection{Clean up the RDP taxon names}

The RDP alignment will have taxon names that most of the downstream software tools will not tolerate because they include special text characters. So, we have 
668

669

written a little Perl script (cleanup.pl) that will remove those special characters and replace them with underscores. This script is included in the zip file of scripts on Figshare [30]. To run cleanup.pl, first move it to your Applications folder. Then, in a Terminal window, navigate to the directory that contains the RDP alignment that you've just downloaded. Then, type or copy/paste:

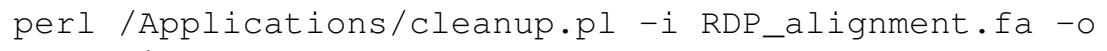

\subsection{Building the Tree with FastTree}

There are two ways to get FastTree, which will be required for building the tree from your alignment. The first, recommended approach, is to use Phylosift (installed in 9.1.4) which contains a working version of FastTree. In this case, you will simply call the program from the Phylosift directory with the following command (be sure the path to Phylosift calls the correct version):

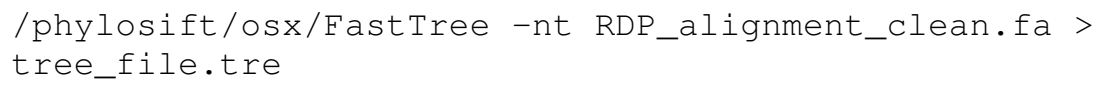

The other option is to install FastTree directly, which is a bit more involved.

Go to http://www.microbesonline.org/fasttree/\#Install and download the FastTree.c program by right clicking on it and saving the link to your Applications folder. To compile the software, navigate to your Applications folder in a Terminal window:

cd /Applications

Then, type or copy/paste: 
696

697

698

699

700

701

703

704

gcc-03-finline-functions -funroll-loops -Wall -o FastTree FastTree.c - lm

This compiling of FastTree requires a software tool called gcc (http://gcc.gnu.org). If your attempt to compile FastTree with the instructions above fails, the most likely reason is that you do not have gcc. You can download and install gcc from Xcode here https://developer.apple.com/downloads/index.action?q=xcode

In order to download Xcode, you will need to register as a developer with Apple which takes only a couple of minutes. After you register, click on the apple next to "Developer" at the top of the page. Then, click on the Xcode download link, which will ultimately take you to the Mac App Store, where you can follow the instructions to install Xcode. Once it is installed, open the program and open preferences (under the Xcode tab). Click on the downloads option and install the command line tools.

Once you have successfully downloaded and installed Xcode and the command line tools, return to your Applications folder in a Terminal window and type or copy/paste again:

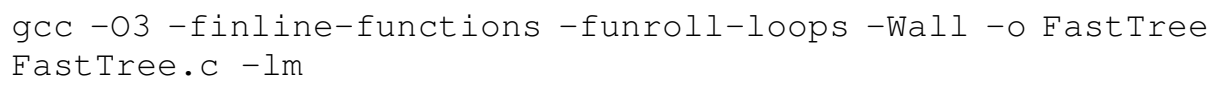

Now, you should have a working version of FastTree. To build your tree, using the cleaned up RDP alignment, type or copy/paste the following (be sure the output name ends in ".tre" to ensure it will be recognized by Dendroscope):

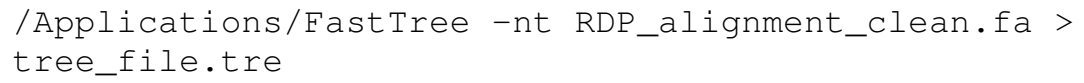




\subsection{Viewing the Tree in Dendroscope}

Download and install Dendroscope.

http://ab.inf.uni-tuebingen.de/software/dendroscope/

You will need to obtain a license here

http://www-ab2.informatik.uni-tuebingen. de/software/dendr

oscope/register/

Enter the license number into Dendrosope and then you can open your phylogenetic tree from the File menu to view it.

Once the tree is visible, the first step is to re-root the tree to the outgroup. Expand the tree by clicking the expansion button (labeled in Figure 11), then scroll through the tree to locate the outgroup. Click on the beginning of the taxon name, to select it, and reroot the tree by going to edit and selecting "re-root".

We recommend viewing the tree as a phylogram, which can be accomplished by clicking on the phylogram button (labeled in Figure 11). From this tree it should be possible to determine the phylogenetic placement of the candidate sequence, and in some cases to give it a name with more certainty than a simple BLAST search. Below are examples of a relatively informative tree and a relatively uninformative tree:

In the tree shown in Figure 12 (genus Brachybacterium), our sample of interest from an assembly is "Brachybacterium muris UCD-AY4" [32]. It falls within a clade where every named member has the same name "Brachybacterium muris", and this name does not occur elsewhere on the tree. Hence, we were confident enough to name our organism as that species. In other words, this sequence falls within a well-supported clade of Brachybacterium muris. 
In the tree shown in Figure 13 (genus Tatumella) our species of interest is Tatumella sp. UCD-D suzukii [20]. In contrast to the Brachybacterium example, here our species falls within a poorly defined clade containing multiple species. In this case, we did not assign a species name to this isolate.

\section{Genome Sequencing}

\subsection{Library Preparation}

The first choice in library preparation is whether to do the library prep yourself or to have the library made by your sequencing provider. The economics of this decision are usually dependent on the number of samples involved. For example, an Illumina TruSeq library prep kit costs around $\$ 2600$ for 48 samples. That's far cheaper than the $\$ 150$ to $\$ 300$ that a typical sequencing provider would charge per sample. However, if you're only preparing a couple of samples there's no reason to buy an entire kit. The requisite time and ancillary consumables and equipment must also be taken into account (see Table 1). Most sequencing facilities offer library preparation services.

\subsection{Kit Options}

Whether you chose to make libraries yourself or use a service provider, the next major choice is of the type of kit. The two most popular choices with Illumina kits are the Nextera transposase-based kits or the TruSeq kits (with or without PCR). These kits are available from Illumina, but there are also comparable options from other vendors (e.g. New England Biolabs and Kapa Bioscience). In addition, there have 
been cost-saving modifications to the Illumina kits, for example [6]. The pros and cons of each type of kit are listed below:

- TruSeq (our recommendation): Pro - The PCR-free protocol minimizes library bias by using mechanical instead of enzymatic DNA fragmentation, and the elimination of PCR results in better assemblies. Con - requires a large amount of DNA (at least $1 \mu \mathrm{g}$ for PCR-free). There is also now a TruSeq LT kit which only requires 100ng of DNA and a reduced number of PCR cycles. This may provide a middle option between PCR-free TruSeq and Nextera.

- Nextera: Pro - It allows for very low amounts of input DNA, down to 1ng in the case of the Nextera XT kit. Con - the transposase has an insertion bias and the extensive PCR required for low input samples will also impact the final assembly [1].

When growing bacteria in culture as described in this workflow, it should almost always be possible to get enough DNA to use PCR-free TruSeq and therefore minimize library preparation biases in the genome assembly.

\subsection{Choosing an insert size}

In our lab, with paired-end 300bp (PE300) reads on the Illumina MiSeq, we target a DNA fragment size (including adapters) of 600-900bp. The high end of the range is constrained by the maximum length of a DNA molecule that can be amplified on the Illumina MiSeq. The low end of the range is defined by the smallest fragment size that will not produce overlapping reads. Ideally, you would sequence only at the high 
791 end of the range because longer insert sizes aid in better genome assembly. However,

792 the range is typically expanded to ensure that enough DNA is available for

793 sequencing. Different sequencing facilities have different opinions on this topic and it

794 is worth having a discussion with your sequencing facility's point of contact before

795 making any libraries. It is very important that all samples have similar insert sizes if

796 multiplexing as described below.

\section{$797 \quad 8.4$ Multiplexing}

798 Coverage (also known as read depth) is the average number of reads representing a

799 given nucleotide. It is a function of the number and size of genomes pooled onto a run

800 and the number and length of reads. The optimal amount of coverage depends on the

801 read length, the assembler being used, and other factors. The capacity of an Illumina

802 MiSeq with PE300 reads is around 15 Gigabases $(\mathrm{Gb})$, which would result in a

803 coverage of $4300 \mathrm{X}$ for a typical bacterium with a $3.5 \mathrm{Mb}$ genome. On the HiSeq with

804 PE125bp reads, this would be over 14,000X coverage. Currently, the recommended

805 coverage for a bacterial genome assembly is $20-200 \mathrm{X}$ depending on the choice of

806 assembler. Therefore, sequencing a single bacterial genome on a full MiSeq or HiSeq

807 run is a significant waste of money and reagents. Furthermore, some current genome

808 assembly algorithms do not perform well given an excess of data, and require

809 down-sampling (i.e., throwing away data, Section 8.6) to achieve the recommended

810 coverage for assembly. We typically multiplex 10-48 genomes on a PE300 MiSeq run

811 and many more on a HiSeq run. If using a kit for library prep, multiplexing is quite

812 straightforward since there are a number of barcoded adapters that come with the kit.

813 We recommend having the sequencing facility demultiplex the samples, as this only

814 requires a list of the barcodes used. 


\section{$815 \quad 8.5$ Collaborate}

816 As described above, current Illumina sequencing systems have much greater capacity

817 than is needed for sequencing a single genome. This means it can be generally

818 beneficial to combine many samples into a single run of a machine. Unfortunately,

819 our experience has been that sequencing facilities will typically not help in the

820 coordination of such pooling of samples (we assume because they do not want to

821 oversee the pooling or deal with the associated accounting hassles). Therefore, it is

822 typically up to the users to carry out such coordination. Though this can sometimes be

823 complicated, it is generally worthwhile, since one can pool together many genomes or

824 metagenomes into a single run of a system and still get enough data for each project,

825 thus making the sequencing cost per project significantly lower. For this to work well,

826 one needs to coordinate the use of barcodes to tag each sample, coordinate the

827 pooling, and have available the informatics required to "demultiplex" samples from

828 each other.

$829 \quad$ 8.6 Downsampling

830 For Illumina data assembled using this workflow, we recommend a coverage of

831 between 20X and 200X. See our more detailed discussion in Section 9.1.3

832 "Interpretation of A5-miseq stats". If you have coverage significantly higher than

$833200 \mathrm{X}$ and wish to downsample your data, we have written a script

834 (sub_sample_reads) for this purpose. Downsampling should not be necessary if

835 following the assembly instructions in this workflow. If downsampling, you will first

836 need to calculate how many reads you want the script to sample. We recommend

837 determining how many reads would be equivalent to $100 \mathrm{X}$ coverage (divide the 
838 genome size by the average read length and multiply by 100). You can download the

839 script from the zipped script file found on Figshare [30]. Create a new directory

840 containing the script (sub_sample_reads) and the reads you wish to downsample.

841 To downsample the data, navigate to the directory you just created (in the

842 terminal) and use the following command

843

844

845

846

847

848

849

850

851

852

853

854

Genome assembly consists of

\subsection{Assembly}

. /subsample_reads.pl <file1> <file2> <\#_reads_to_keep> <output_file_name>

for example

./subsample_reads.pl test_1.fq test_2.fq 250 my_reads.fastq

For further directions and documentation you can view the script on github

\section{Genome Assembly and Annotation}

857 2. error correction

858 3. contig assembly

859 4. scaffolding (optional) 


\section{5. verification of scaffolds/contigs}

The first step simply removes poor quality sequences, as well as adapter sequences left over from sequencing. Some assemblers follow this with error correction where reads are compared to each other to eliminate sequencing errors. Next is contig assembly where overlapping reads are assembled into long continuous stretches of sequences. Scaffolding refers to the alignment and orientation of these contigs relative to each other (where possible). The last step is verification where reads are mapped back to the contigs/scaffolds to reduce misassemblies.

There is a plethora of programs that can perform some, or most of these steps. These programs include commercial and open-source options, some are very user friendly and some are extremely difficult to use/install. Common assemblers for bacterial genomes include SPAdes [5], MIRA [9], SGA [40], Velvet [44] CLC (CLC Bio), and A5 [43]. Good sources for overviews of genome assemblers and the assembly process include the GAGE project [37], the GAGE-B project [33], and the Assemblathon Project [21].

In this workflow, we recommend use of the open source A5 assembly pipeline which automates all of the steps described above with a single command [43]. A5 is designed to work with raw, demultiplexed Illumina data and a recent version (A5-miseq) has been optimized for longer reads from the MiSeq [11]. Input files should have the .fastq extension. See (http://en.wikipedia.org/wiki/FASTQ_format) for a description of the fastq format. You will need one of the two following (per genome): 1) a single .fastq file that contains both forward and reverse reads, or 2) two .fastq files, one with forward reads and one with the corresponding reverse reads. These fastq files can optionally be gzip compressed (as indicated by the .gz file name 
884 extension). You may need assistance from your sequencing center in locating and 885 accessing these files.

886 Download/Install A5 from

887 http://sourceforge.net/projects/ngopt/

$888 \quad$ Follow the (expert) instructions located

889 http://sourceforge.net/projects/ngopt/files/?source=navba

$890 r$

$891 \quad$ or

$892 \quad$ Follow a video made by David Coil

893 https://www. youtube. com/watch?v=Ad6HJevC5U8

$894 \quad$ or

$895 \quad$ Follow these instructions:

896 After downloading and unzipping the program, change the name of the folder to

897 a5_pipeline and move it from your downloads folder to your Applications folder.

898 Then, create a new folder which will contain the files generated by the pipeline on

899 your Desktop. By the way, there's nothing special about having your file on the

900 Desktop, it's just there to simplify our instructions. We will refer to this folder as

901 “a5_output”, but you should use a more informative name.

$902 \quad$ 9.1.1 Running A5-miseq

903 Open a Terminal window and navigate to a5_output. A5-miseq will write all of the

904 assembly output files to the same folder from which you run the program. In this 
example, the newly created folder is on the Desktop and named a5_output so the syntax for navigating to the folder in a Terminal window is

cd Desktop/a5_output/

Now that you are in the folder where you want your genome assembly to appear, you are ready to run the program. First, type or copy/paste (don't hit return yet, and don't copy a carriage return!):

/Applications/a5_pipeline/bin/a5_pipeline.pl

Then, drag and drop in the input file(s) into the same Terminal window (or type the path to them if you know it). Finally, type a name that will be included in all of all of your output files. So, your command line should look like this:

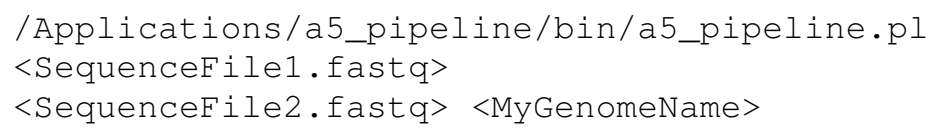

The program may take a few hours to run. Once it is completed, the terminal will display "Final assembly in MyGenome.final.scaffolds.fasta". The complete assembly will be located in the a5_output folder.

Among the numerous files generated by A5, two of particular importance are the "MyGenome.contigs.fasta" and "MyGenome.final.scaffolds.fasta" which contain the contigs and scaffolds, respectively.

In addition, A5-miseq generates a file containing information about the quality of the assembly called "MyGenome.assembly_stats.csv" (see Section 9.1.3 for interpretation). 


\subsubsection{Assembly Validation}

934 There are three components to genome assembly validation. The first is the overall

935 "quality" of the assembly, assessed by examining the stats provided by A5-miseq

936 (discussed below). The second is verification that the organism sequenced is the

937 organism of interest, simply by checking the assembled $16 \mathrm{~S}$ rDNA sequence using a

938 BLAST search (see Section 6 above). The third is "completeness," which is difficult

939 to measure without a closely-related reference genome. Nevertheless, we can get an

940 idea of how complete the genome is by looking for highly conserved "housekeeping"

941 genes that are found in almost every bacterial genome using a program called

942 PhyloSift [15] (see Section 9.1.5).

\section{$943 \quad$ 9.1.3 Interpretation of A5-miseq stats}

944 Open "MyGenome.assembly_stats.csv" in Excel. The first two numbers, shown in 945 columns 2 and 3, are the number of contigs and scaffolds. Defining a "good" or "bad"

946 assembly starts here. A finished assembly would consist of a single contig with no

947 unresolved nucleotides but that is extremely unlikely to result from short read data. At

948 the other extreme, we would consider a bacterial assembly in 1000 contigs to be very

949 fragmented. In our experience, acceptable bacterial assemblies using Illumina PE300

950 data, assembled with A5, tend to range from 10-200 contigs. It is also worth noting

951 that unless studying genomic organization, the number of contigs is less important

952 than the gene content recovered by the assembly which is typically $>99 \%$ using

953 A5-miseq [11].

954 "Genome Size" and "Longest Scaffold" are represented in base pairs. While 955 genome size can vary within taxa, this can be a second useful sanity check for the 
assembly. When expecting a 5MB genome based on other sequenced isolates from the same genus, if the assembled genome size is $2 \mathrm{MB}$ or $10 \mathrm{MB}$, a red flag should be raised. "N50" represents the contig size at which at least $50 \%$ of the assembly is contained in contigs of that size or larger. This metric, combined with the number of contigs is the most common measure of assembly quality; larger is better. An N50 of $5,000 \mathrm{bp}$ would be quite poor, meaning that half of the entire assembly is in contigs smaller than 5,000bp. On the other hand an N50 of 1,000,000bp is considered very good for bacterial genomes sequenced with Illumina technology.

\section{The number of raw reads/raw nucleotides "Raw reads"/"Raw nt" and} error-corrected reads/nucleotides "EC Reads"/“EC nt" counts are useful for seeing what percentage of the data have been discarded. A very large difference between these numbers (“\% reads passing EC"/“\% nt passing EC") would indicate either poor quality sequence data or significant adapter contamination. Adapter contamination rates can be high when the insert size is too small or if there were problems during library preparation. Poor quality sequence data can result from loading the libraries at a molar concentration that was too high for the instrument, from mechanical issues preventing focus of the sequencing instrument's cameras, or from use of a compromised batch of sequencing reagents. Resolution of these issues would entail a discussion with your sequencing provider.

A5-miseq reports three depth of coverage statistics which can be used to assess whether sufficient data have been collected for genome assembly. First is the "Raw cov" which is simply the total number of base pairs of sequence data, divided by the assembly size. This gives an estimate of the average number of reads covering each base in the assembly. The actual number of reads at each site can and will vary substantially from the average. The second statistic is the "Median cov" which gives 
981 the median depth of coverage among all sites in the assembly. That is, $50 \%$ of sites

982 will have greater coverage and $50 \%$ will have less than this value. "10th percentile

983 cov" indicates a coverage level below which only $10 \%$ of sites in the assembly fall.

984 For Illumina data, the ideal median coverage will lie between $\sim 20 \mathrm{X}$ and 100X. If you

985 have much less than $20 \mathrm{X}$ median coverage, the quality of individual base calls may

986 be compromised. Ideally, the 10th percentile coverage will be higher than 10 , for

987 similar reasons.

988 A separate metric of the base call quality is also reported by A5-miseq as "bases

989 > $=$ Q40”. Following assembly, A5-miseq realigns the reads to the assembled

990 sequence and estimates the accuracy of the nucleotide called at each site in the

991 assembly. These accuracies are provided as PHRED quality scores [25], which

992 represent log-scaled probabilities of accuracy. For example a PHRED score of 20

993 (Q20) indicates a 99\% chance of the correct base, while Q30 and Q40 indicate 99.9\%

994 and $99.99 \%$ probabilities of the correct base being called. A5-miseq reports the

995 number of assembly bases called with at least Q40.

$996 \quad$ 9.1.4 Verification of 16S Sequence

997 Follow the steps described in Section 9.2.3, then Section 6, to obtain the 16S rDNA

998 sequence from the assembly and verify that what you sequenced is what you were

999 expecting.

$1000 \quad$ 9.1.5 Assessing Completeness with Phylosift

1001 PhyloSift: Navigate to http://phylosift.wordpress.com

1002 Download and unzip the latest version of Phylosift 
./phylosift search /Users/microBEnet/Desktop/Data-Genomes/

1014 so it may take a few minutes.

1015 From the PhyloSift directory Move to the "PS_temp" directory

1016 Within this directory, Phylosift has created a directory with the same name as the

1017 input file. Move (cd) to this new directory, and then move to "blastDir".

1018 Open the marker_summary.txt file in the blastDir directory.

1019

1020

1021

1022

1023

1024

1025

1026

1027

1028 less marker_summary.txt

The DNGNGWU0001-00040 markers represent 37 highly conserved bacterial genes, if one is missing it won't show up as a zero, it is necessary to manually verify the list. Most of the genes should only appear once. An occasional 2 is fine, but if all/a majority of the genes appear twice or even three times you have most likely sequenced multiple bacteria together. Additionally, check to make sure there is no 18S RNA sequence (at the top of the list) to ensure your sample has not been contaminated with a eukaryote (e.g. yeast). 
1030 analysis so do not be concerned if they are not listed. Conversely, Marker 13 is

1031 sometimes present in multiple copies and this is not a cause for concern.

\section{$1032 \quad 9.2$ Annotation}

\section{$1033 \quad$ 9.2.1 Options}

1034 Genome annotation is the process of predicting genes within a genome sequence and

1035 attempting to assign function to those genes based on homology to known sequences.

1036 Note that we are not describing a genome "analysis" here. While genome annotation

1037 marks the final step in our workflow, it is just the beginning of a thorough genome

1038 analysis. We recommend performing this step as the bare-minimum required to

1039 include a very basic description of the genomic content for a genome publication.

1040 There are a number of different pipelines available for the annotation of bacterial

1041 genomes. These include Prokka [39], IMG [34], RAST [35], GLIMMER [16], PGAP

1042 [3] and others.

1043 Each of these pipelines has advantages and disadvantages, and each will give

1044 slightly different results. Here we recommend RAST since it is web-based, easy to

1045 use, returns results within hours, and provides a convenient toolbox for analyzing the

1046 results. However, RAST annotations are very difficult to submit to NCBI so we

1047 recommend allowing NCBI to re-annotate the genome with PGAP upon submission.

1048 Also, we recommend reporting the annotation results from the PGAP annotations in

1049 the genome publication (for consistency). 


\subsubsection{RAST Annotation}

1051 Navigate to http://rast.nmpdr.org/ and register a new account. Once you

1052 have created an account, log in. Hover over the "Your Jobs" tab at the top of the page

1053 and click on "Upload New Job". In order to proceed you must specify a domain, a

1054 genus, a species, and the genetic code (usually "11"). Click "Finish the Upload."

1055 The annotation will take some time, ranging from 2 hours to a few days,

1056 depending on server load. RAST will email you when it is complete. Once the

1057 annotation is complete, use their SEED Viewer to explore the annotation and

1058 metabolic pathways of the organism. From the RAST results, you can obtain

1059 information such as the presence or absence of a particular gene/pathway and you can

1060 compare the annotation to other genomes in their database.

$1061 \quad$ 9.2.3 Obtain the Full-Length 16S Sequence from the Assembly

1062 (Skip this step if you are building the tree using the 16S rDNA sequence from Sanger 1063 sequencing)

1064

1065 1. Go to RAST and sign in

1066 2. On the "Jobs Overview" page, click on "view vetails" (under annotation

1067 progress) for the microbe you are working with.

1068 3. Click on "Browse annotated genome in SEED viewer" (At the top of the

1069 page)

1070 4. Click on "Browse through the features of [organism name]" 
1071

1072

1073

1074

1075

1076

1077

1078

1079

1080

1081

1082

1083

1084

1085

1086

1087

1088

1089

1090

1091

5. In the Function column, search for "ssurna" or "SSU rRNA" (if it doesn't work at first then refresh the page)

6. Find the ssuRNA that is $1400-1800$ bp in length (often Illumina assemblies also have fragments of $16 \mathrm{~S}$ rDNA sequence that are only a few hundred base pairs long)

7. In the Feature ID column, click on the link for the sequence with the correct length

8. Click on the "Sequences" tab (around the middle of the page )

9. Click on "Show Fasta"

10. Click on "Download Sequences" and save as a fasta file. Rename the file to something informative.

11. Double check the identity of the sequence at BLAST:

http://blast.ncbi.nlm.nih.gov/Blast.cgi?PROGRAM=blas tn $\backslash \& P A G E \_T Y P E=B l$ ast Search $\backslash \& L I N K \_L O C=$ blasthome

\section{Data Submission}

This section describes how to submit contigs and scaffolds (if applicable) as a Whole Genome Shotgun (WGS) submission to Genbank. We also recommend allowing NCBI to annotate the genome, since submitting RAST annotations to Genbank can be prohibitively complicated. The genomes are automatically shared with the DNA Data Bank of Japan (DDBJ) and the European Molecular Biology Laboratory (EBML). In addition, genomes from Genbank are automatically pulled into the Integrated 
1092 Microbial Genomes (IMG) database hosted at the Joint Genome Institute (JGI), and

1093 are annotated there as well. This section also describes how to submit the raw reads,

1094 in this case we use the European Nucleotide Archive (ENA) for ease of use but the

1095 reads will be automatically incorporated into the Short Read Archive (SRA) at NCBI

1096 as well.

1097 Before going any further you must decide if you are submitting contigs or

1098 scaffolds. Because recent versions of A5 have very good contig generation, often

1099 scaffolding doesn't prove much additional information. For example a genome with

110035 contigs in 30 scaffolds should probably be submitted as contigs only. Submitting

1101 scaffolds is significantly more complicated than submitting contigs, instructions for

1102 both are given below.

$1103 \quad \mathbf{1 0 . 1}$ Submitting contigs only

1104 Use this section if submitting only contigs, presumably in .fasta format

1105 Navigate to http://www.ncbi.nlm.nih.gov. Create an account and/or

1106 login. Then, create a BioProject at NCBI by navigating to

1107 https://submit.ncbi.nlm.nih.gov/subs/bioproject/ and clicking

1108 on "New submission." Fill in the personal information for the submitter.

1109 Below, in italics, are the responses that we typically give for a genome

1110 sequencing project.

$1111 \quad$ Project type

1112 
$\begin{array}{lll}1113 & \text { - } & \text { Project data type-genome sequencing } \\ 1114 & \text { • } & \text { Sample scope-monoisolate } \\ 1115 & \text { - } & \text { Material-genome } \\ 1116 & \text { - Capture-whole } \\ 1117 & \text { - Methodology-sequencing } \\ 1118 & \text { - Objective-assembly } \\ 1119 & \text { Target }\end{array}$

1120

1121 - Organism Name

1122 - If you have other information feel free to add it

$1123 \quad$ General info

1124

1125 - We recommend choosing Release immediately following curation

1126 - Project Title

1127 - Public Description

1128 - Relevance-Environmental

1129 - Biosample-blank

1130 - Publications-blank 
1131 Once the project is submitted, refresh the page and copy down the Bioproject ID

1132 (it starts with "PRJNA")

\section{$1133 \quad \mathbf{1 0 . 2}$ Create a Whole Genome Shotgun (WGS)}

\section{Submission}

1135 Navigate to https://submit.ncbi.nlm.nih.gov/subs/wgs/

1136 Click on the "New Submission" button at the top, fill in your information, and

1137 click "Continue".

$1138 \quad$ General Info

1140 - BioProject-Yes, add the BioProject identification sequence (from the

$1141 \quad$ BioProject submission, starts with PRJNA)

1142 - Biosample-No

1143 - Release date-Optional but we recommend Release immediately following

$1144 \quad$ curation

1145 Do not check the box stating, "Genome assembly structured comment is in the 1146 contig .sq file"

1147

1148 - Assembly Method-Choose other, fill in the blank with A5 Assembly Pipeline

1149 (version can be found in the asssembly_stats.csv file) 
1150 - Version or date program was run - a5-miseq-macOS-20140521

1151 - Assembly name - give your assembly an appropriate name

1152 - Genome coverage- this is provided in the output from A5

1153 - Sequencing technology - Illumina (Miseq or HiSeq)

1154 - Is this the full representation of the genome? Yes

1155 - Is this the final version? Yes

1156 - Do you intend to annotate this version? No

1157 - Is it a part of a multiisolate project? No

1158 - Is it a de novo assembly? Yes

1159 - Is it an update of existing submission? For most projects the answer to this

$1160 \quad$ will be $N o$

1161 - BioSample Type: Microbe

$1162 \quad$ BioSample attributes

1163

1164 - Sample Name

1165 - Organism

1166 - Strain

1167 - Collection date

1168 - Geographic location 
1169

1170

1171

1172

1173

1174

1175

1176

1177

1178

1179

1180

1181

1182

1183

1184

1185

1186

1187

1188

1189

1190

- Isolation source

- Files

- Select We have files for traditional split contigs OR gapped sequences

- Select _ "FASTA", upload the files

- Select "No" for the question about scaffolds

• "Is any sequence a complete chromosome?" No

- "Does any sequence belong to a plasmid" No

-Check the box below to annotate this prokaryotic genome in the NCBI

prokaryotic annotation pipeline before being released. This will allow NCBI to use

their PGAP pipeline to annotate the genome, and this annotation will be automatically attached to the project.

Click "Submit" and you're done! You will receive a series of e-mails from NCBI confirming your submission and notifying you of any problems. Once the submission is pre-processed you'll get an accession number. Note however that the data will not be released until final processing. The accession number is not acceptable for publication until after the final release of the data.

Potential problems with data submission:

Sometimes contigs that are submitted belong to contaminating organisms, or to the PhiX that is often used in sequencing. If this is the case, you will receive an email from NCBI telling you which contigs to remove. It's important to note that after removing contigs, you need to rename all of your remaining contigs so as to not be missing numbers in the sequence. Below is a simple command that renumbers the 
1191 contigs in the cleaned file (the original file with the contaminated contigs removed)

1192 and saves them to a new file (test.fa is the name of your cleaned file and test 2 .fa is the

1193 name you want the renumbered file to have):

1194

1195

1196

1197

\section{$1198 \quad \mathbf{1 0 . 3}$ Submitting scaffolds}

1199 Only use this section if you are submitting scaffolds, in most cases assembly with A5

1200 will render this step unnecessary. Many of the steps are the same as for submitting

1201 contigs, only the differences are shown here.

1202 Before submitting your scaffolded genome, you will need to have available 4-5

1203 files which are listed below.

1204 File types used in data submission:

1205

1206 - AGP file (.agp). This is a file required by NCBI to describe scaffolding

1207 - FASTA file (.fasta). This is the standard file type for sequence data, produced

$1208 \quad$ in this case by A5-miseq

1209 - FSA file (fsa). Same as a FASTA file but with a different extension

1210 SQN file (.sqn). The file type for sequence data required by NCBI

1211 - SBT file (.sbt). This is a template file type used by NCBI 
1212 FASTA2AGP First, create the agp file. In the terminal, navigate to the directory

1213 containing your scaffolds file. Run the fasta2agp.pl script included with A5 on the

1214 scaffold file output by the A5 assembly "my_scaffolds.fasta".

$1215 \quad$ Syntax is:

1216

1217

1218

1219

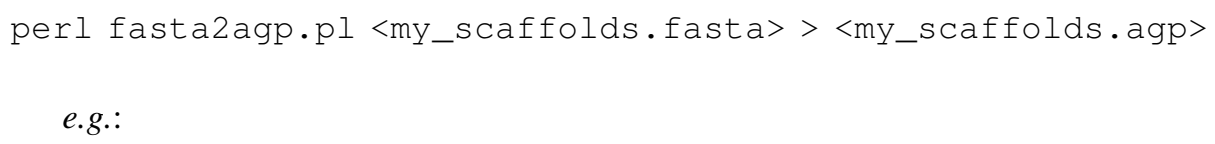

1220

1221

1222

1223

1224

1225

1226

1227

perl

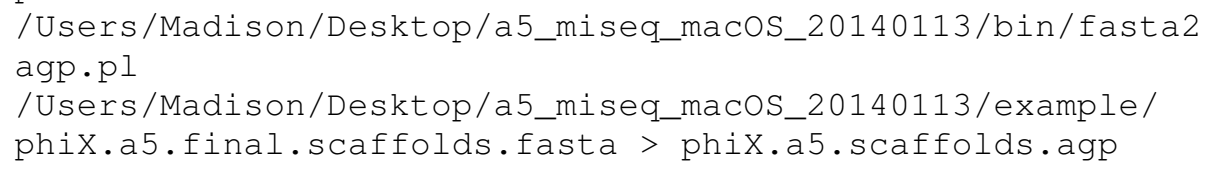

If this runs successfully then you should see a both the .fsa and agp files in your current directory.

1230 information" in a contig, not a gap between contigs (whereas A5 has no minimum

1231 gap size). Therefore, NCBI requires that contigs separated by less than 10 nucleotides

1232 be merged. This script performs that merging, meaning that the number of contigs in

1233 the .fsa file may be less than in your input file. Therefore, we recommend counting

1234 the contigs in the .fsa file:

1235 To count them in the terminal use the syntax

1236

1237

1238

1239

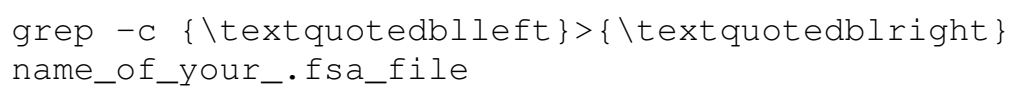


1240 Important Note: If after running the fasta2agp.pl script and counting the contigs

1241 you have the same number of contigs as starting scaffolds, then you submit only the

1242 contigs as described in Section 10.1.

1243 Create a SBT template Create a SBT template file at NCBI

1244 http://www.ncbi.nlm.nih.gov/Websub/template.cgi The

1245 BioProject \# is the Bioproject ID starting with "PRJNA" which you received above.

1246 BioSample can be left blank.

1247 When you click "Create the template", it will automatically download to your

1248 computer as template.sbt. We recommend immediately renaming the file to the

1249 appropriate project.

1250 Tbl2asn Download the tbl2asn program from

1251 ftp://ftp.ncbi.nih.gov/toolbox/ncbi_tools/converters/by_p

1252 rogram/tbl2asn/.

1253 If you are using Safari, a window will pop up asking for login information, just

1254 choose guest and unzip the version of the program that is compatible with your

1255 operating system. Other browsers will take you to a page with a lot of tbl2asn

1256 programs, download the one compatible with your operating system.

1257 After downloading the desired command-line program, double click to

1258 uncompress the archive and rename the resulting file to tbl2asn. Now change the file

1259 permissions of the file (in the terminal) since transfer by FTP resets the permissions.

$1260 \quad$ Syntax is:

1261

1262

1263

chmod 755 tbl2asn 
1264 Once you have changed the permissions, create a new directory and place tbl2asn

1265 along with the .sbt file and .fsa files into the folder.

1266 Run the tbl2asn program using the following syntax. You will need to fill out the 1267 organism name, strain, location, collection date, and isolation source specific to your 1268 own project.

1269

1270

1271

1272

1273

1274

1275

1276

1277

1278

1279

1280

1281

1282

1283

1284

1285

1286

1287

1288

1289

1290

1291

1292

1293

1294

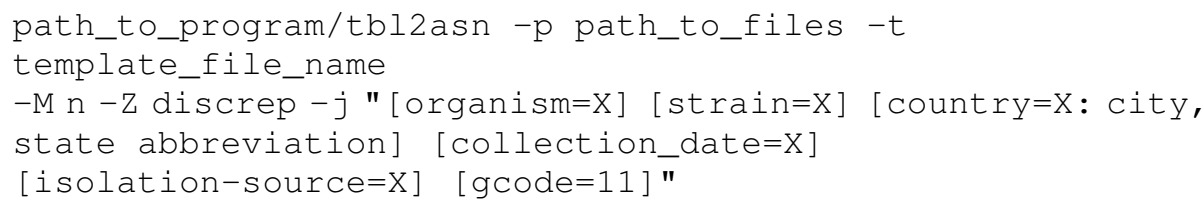

Following the $-\mathrm{p}$ is the path to the directory containing the fsa file, following the $-\mathrm{t}$ is the path to and name of the .sbt template file

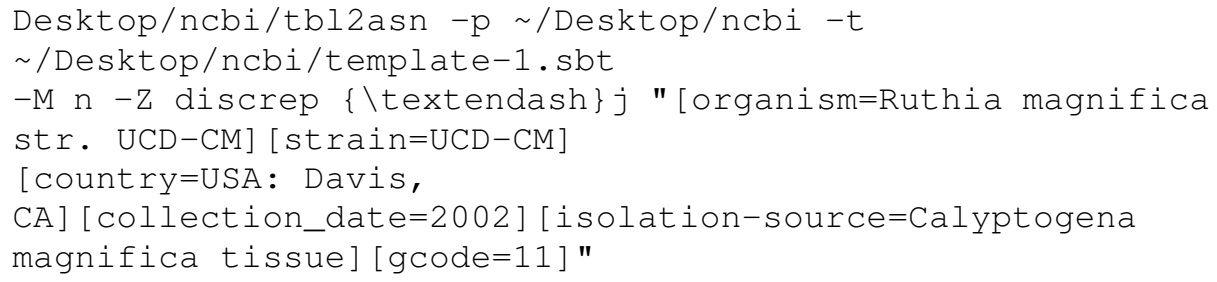

The program will output the necessary files into the directory you created earlier

(ensure no errors were generated by opening the errorsummary.val file and making sure it is blank, or listing the directory contents $(\$ 1 \mathrm{~s}-\mathrm{lh})$ to ensure it has zero bytes)

Once these files are created, submission is similar to that for contigs. However, you will have to specify that you are using scaffolds and to upload the agp file in addition to the .sqn file. 


\section{Submitting Raw Reads to ENA/SRA}

1296 We recommend using Safari or Firefox for this step, in our hands Chrome can

1297 have issues with the Java requirements for uploading files.

1298 Go to https://www.ebi.ac.uk/ena/about/sra_submissions and

1299 create an account.

1300 Successful creation of an account should take you to the "Welcome to ENA's

1301 Sequence Read Archive (SRA) Webin submission system” screen.

1302 Click on "New Submission" tab.

1303 Select "Submit sequence reads and experiments".

1304 Click on "Data Upload Instructions" towards bottom of page.

1305 This takes you to a variety of options for uploading files depending on your

1306 preference and operating system. We use the Webin Data Uploader. Click on the link

1307 which will download a .jlnp file. Open and run this file. Depending on your system

1308 you may have to download and install a new version of Java. On some systems you

1309 may have to right-click the .jlnp file and open with "Java Web Start".

$1310 \quad$ Login using your email address and password.

1311 In the WebinDataUploader, in the blank area to the right of the Local Upload

1312 directory, navigate to the directory on your computer containing the reads (using the

1313 path as you would in the terminal).

1314 Select the file(s) containing the reads and click "Upload". 
1315 (Note that paired-end data is required to be in two separate fastq files. If your

1316 data came as one interleaved file, then the separated fastq files can be found in the

1317 directory where the A5 assembly was performed as [project name].raw1_p1.fastq.gz

1318 and [project name].raw1_p2.fastq.gz )

1319 Note that the only acceptable file types for submission are gzip (.gz) and bzip

1320 (.bz2). To gzip files in the Terminal use the following syntax:

1321

1322

1323

1324

gzip [filename]

1325 submission system) and select the "Next" button. During this process, refreshing the

1326 page or navigating away from the page will reset the form and the information will be

1327 lost.

1328 Click "Create a New Study". Fill in descriptions of the project and proceed to 1329 next tab. Select the appropriate metadata format, or in most cases the ENL default

1330 sample checklist at the bottom. Note that the default release date is three months from

1331 the current date, change this if the data should be released sooner.

1332 You should now be at the "Sample" page. Required fields are listed on the right

1333 and optional additional fields can be selected from the options on the right. Fill out

1334 the appropriate fields and click on "Next".

1335 Note: If you are submitting data for an organism that doesn't have a Taxon ID

1336 (“Tax ID”) then you need to e-mail ENA to receive one (datasubs@ebi.ac.uk). If you

1337 have already submitted the genome to NCBI then you can retrieve the Taxon ID from

1338 your BioProject page there. On the ENA page, you will be able to search for the 
1339 Taxon ID and find your organism under the "Organism Details" tab but you won't be

1340 able to find it using the name of the organism.

1341 On the "Sample" page, click the "+ Add" button under sample group details. Fill

1342 in the unique name under basic details, add the Tax ID if it wasn't added previously,

1343 and click "Next". On the "Run" page, select the appropriate data type. Fill in the

1344 required fields (they change with data type).

1345 Note: "Insert size" cannot be a range, only a number. With our 600-900bp

1346 libraries, we enter 750 here.

1347 Click "Submit" and confirm submission. You will immediately receive a

1348 confirmation email but it takes some time before the information is actually live at the

1349 ENL links.

\section{Publication}

1351 While submitting reads and an assembly to NCBI/ENA as described above makes the

1352 data accessible to the world, a little more effort will put that data into a more useful

1353 context. There are two general types of publications that describe microbial genomes.

1354 "Data papers" focus largely or entirely on describing the generation of the sequence,

1355 assembly, and (sometimes) annotation. "Analysis papers" dive much deeper into the

1356 genome and what the genome may reveal about the biology, evolution and ecology of

1357 the organism and its relatives. The latter (analysis papers) is outside the scope of this

1358 workflow, but a brief discussion of options for the former (data papers) is warranted.

1359 Several options exist for relatively short publications that simply describe the 
1360 generation of a genome sequence. Note that all of these publications require accession

1361 numbers for the data, as described in the section on data submission above.

1362 Our lab often publishes genome sequence "data papers" in "Genome

1363 Announcements", a open-access journal published by the American Society for

1364 Microbiology (ASM). These papers are no more than 500 words in length, no figures

1365 are allowed, and they are not formally peer-reviewed (they do go through review by

1366 an editor). This format allows information about the generation of a genome sequence

1367 to enter the publication record with a minimum of effort.

1368 "Marine Genomics" is an option for marine-associated microbial genome papers

1369 (“Genomics/Technical Resources”). The peer-reviewed journal is not open-access

1370 (but has that option), allows for up to 3 figures and 1000 words but does require

1371 deposition of the strain in a culture collection.

1372 "Standards in Genomic Sciences (SIGS)" is a peer-reviewed open-access

1373 publication with a "Short Genome Report" option. These reports adhere to a

1374 standardized format and are significantly longer than those of the previously listed

1375 options.

1376 Another choice is the closed-access "FEMS Microbiology Letters" (which has an

1377 open-access option). This journal has a non peer-reviewed "Genome Announcement"

1378 article type which does allow figures.

1379 For microbes associated with the gut, the open-access "Gut Pathogens" has a

1380 "Genome Announcement" article type. These articles are peer-reviewed and limited

1381 to two figures and 20 references. 


\section{Discussion}

1383 In an effort to demystify the process of microbial genome sequencing and de novo

1384 assembly, we have designed a workflow that would allow a small lab, one operating

1385 without a specialized technician or bioinformatician, to take a sample from swab to

1386 genome publication. There are many options for sequencing, assembling, and

1387 annotating microbial genomes. This workflow is only one path through the numerous

1388 choices that could be made in a genome sequencing project. All of the scripts and

1389 programs for this workflow are open-source and available online for free to ensure

1390 that individual researchers and small groups are able to access and utilize the tools

1391 necessary to complete the workflow.

1392 Sequencing, sharing, and publishing a genome sequence can certainly be

1393 considered as an important process in its own right. Once a genome is shared, other

1394 people can use that genome for various purposes. However, just because one can stop

1395 after publishing and releasing a genome sequence that does not mean one should

1396 ignore what else one can do with the data. A genome sequence is also a starting point

1397 for many computational and laboratory analyses that can provide insight into

1398 evolution, ecology, physiology, biochemistry, metabolism, and more. Such analyses

1399 are beyond the scope of this workflow and paper but that should not be taken as

1400 implying they are not interesting, useful or important.

\section{$1401 \quad 13$ Acknowledgements}

1402 The authors would like to thank the many people who contributed to this workflow by

1403 field-testing various sections; Makayla Betts, Camilla Dayrit, Andrew Stump, 
1404 Muntaha Samad, Henna Hundal, Cassie Ettinger, and Hannah Holland-Moritz.

1405 Additionally the authors would like to thank Authorea for technical assistance with 1406 their article platform. Funding for this project was provided by the Alfred P. Sloan

1407 Foundation through a grant to J. A. Eisen as part of their "Microbiology of the Built

1408 Environment" program.

\section{References}

1410 [1] Daniel Aird, Michael G Ross, Wei-Sheng Chen, Maxwell Danielsson,

1411 Timothy Fennell, Carsten Russ, David B Jaffe, Chad Nusbaum, and Andreas

1412 Gnirke. Analyzing and minimizing PCR amplification bias in Illumina

1413 sequencing libraries. Genome Biology, 12(2):R18, 2011.

1414 [2] S Altschul. Basic Local Alignment Search Tool. Journal of Molecular

1415 Biology, 215(3):403-410, Oct 1990.

1416 [3] Samuel V. Angiuoli, Aaron Gussman, William Klimke, Guy Cochrane,

1417 Dawn Field, George M. Garrity, Chinnappa D. Kodira, Nikos Kyrpides,

1418 Ramana Madupu, Victor Markowitz, and et al. Toward an Online Repository of

1419 Standard Operating Procedures (SOPs) for (Meta)genomic Annotation. OMICS:

1420 A Journal of Integrative Biology, 12(2):137-141, Jun 2008.

1421 [4] Sandra L. Baldauf. Phylogeny for the faint of heart: a tutorial. Trends in 1422 Genetics, 19(6):345-351, Jun 2003.

1423 [5] Anton Bankevich, Sergey Nurk, Dmitry Antipov, Alexey A. Gurevich,

1424 Mikhail Dvorkin, Alexander S. Kulikov, Valery M. Lesin, Sergey I. Nikolenko, 1425 Son Pham, Andrey D. Prjibelski, and et al. SPAdes: A New Genome Assembly 


\begin{tabular}{|c|c|}
\hline 1426 & Algorithm and Its Applications to Single-Cell Sequencing. Journal of \\
\hline 1427 & Computational Biology, 19(5):455-477, May 2012. \\
\hline 1428 & [6] Michael Baym, Sergey Kryazhimskiy, Tami D. Lieberman, Hattie Chung, \\
\hline 1429 & Michael M. Desai, and Roy Kishony. Inexpensive Multiplexed Library \\
\hline 1430 & Preparation for Megabase-Sized Genomes. Technical report, jan 2015. \\
\hline 1431 & [7] Z. A. Bendiks, J. M. Lang, A. E. Darling, J. A. Eisen, and D. A. Coil. Draft \\
\hline 1432 & Genome Sequence of Microbacterium sp. Strain UCD-TDU (Phylum \\
\hline 1433 & Actinobacteria). Genome Announcements, 1(2):e00120-13-e00120-13, Mar \\
\hline 1434 & 2013. \\
\hline 1435 & [8] Jacqueline Z-M Chan, Mihail R Halachev, Nicholas J Loman, Chrystala \\
\hline 1436 & Constantinidou, and Mark J Pallen. Defining bacterial species in the genomic \\
\hline 1437 & era: insights from the genus Acinetobacter. BMC Microbiology, 12(1):302, \\
\hline 1438 & 2012. \\
\hline 1439 & [9] B. Chevreux. Using the miraEST Assembler for Reliable and Automated \\
\hline 1440 & mRNA Transcript Assembly and SNP Detection in Sequenced ESTs. Genome \\
\hline 1441 & Research, 14(6):1147-1159, May 2004. \\
\hline 1442 & Andrew C Clarke, Stefan Prost, Jo-Ann L Stanton, W Timothy J \\
\hline 1443 & White, Matthew E Kaplan, and Elizabeth A Matisoo-Smith. From cheek swabs \\
\hline 1444 & to consensus sequences: an A to Z protocol for high-throughput DNA \\
\hline 1445 & sequencing of complete human mitochondrial genomes. BMC Genomics, \\
\hline 1446 & $15(1): 68,2014$. \\
\hline
\end{tabular}


[13] David Coil;. From Swab to Publication Sample Data (Tatumella). http://dx.doi.org/10.6084/m9.figshare.1064368, 2014.

J. R. Cole, Q. Wang, J. A. Fish, B. Chai, D. M. McGarrell, Y. Sun, C. T. Brown, A. Porras-Alfaro, C. R. Kuske, and J. M. Tiedje. Ribosomal Database Project: data and tools for high throughput rRNA analysis. Nucleic Acids Research, 42(D1):D633-D642, nov 2013.

Aaron E. Darling, Guillaume Jospin, Eric Lowe, Frederick A.

Matsen, Holly M. Bik, and Jonathan A. Eisen. PhyloSift: phylogenetic analysis of genomes and metagenomes. PeerJ, 2:e243, Jan 2014.

[16] A. L. Delcher, K. A. Bratke, E. C. Powers, and S. L. Salzberg. Identifying bacterial genes and endosymbiont DNA with Glimmer. Bioinformatics, 23(6):673-679, Mar 2007.

[17] A. L. Diep, J. M. Lang, A. E. Darling, J. A. Eisen, and D. A. Coil. Draft Genome Sequence of Dietzia sp. Strain UCD-THP (Phylum Actinobacteria). Genome Announcements, 1(3):e0197-13-e00197-13, May 2013. 
1470

1471

1472

1473

1474

1475

1476

1477

1478

1479

1480

1481

1482

1483

1484

1485

1486

1487

1488

1489

1490

1491

1492
[18]

M. Drancourt and D. Raoult. Sequence-Based Identification of New

Bacteria: a Proposition for Creation of an Orphan Bacterium Repository.

Journal of Clinical Microbiology, 43(9):4311-4315, Sep 2005.

M. I. Dunitz, P. M. James, G. Jospin, J. A. Eisen, D. A. Coil, and

J. A. Chandler. Draft Genome Sequence of Tatumella sp. Strain UCD-Dsuzukii

(Phylum Proteobacteria) Isolated from Drosophila suzukii Larvae. Genome

Announcements, 2(2):e00349-14-e00349-14, Apr 2014.

[20]

MI Dunitz, PM James, G Jospin, JA Eisen, DA Coil, and

JA Chandler. Draft Genome Sequence of Tatumella sp. Strain UCD-D suzukii

(Phylum Proteobacteria) Isolated from Drosophila suzukii Larvae. Genome

Announc, 2, Apr 2014.

D. Earl, K. Bradnam, J. St. John, A. Darling, D. Lin, J. Fass,

H. O. K. Yu, V. Buffalo, D. R. Zerbino, M. Diekhans, and et al. Assemblathon

1: A competitive assessment of de novo short read assembly methods. Genome Research, 21(12):2224-2241, Dec 2011.

R. C. Edgar. MUSCLE: multiple sequence alignment with high accuracy and high throughput. Nucleic Acids Research, 32(5):1792-1797, Mar 2004.

[23] David J Edwards and Kathryn E Holt. Beginner's guide to comparative bacterial genome analysis using next-generation sequence data. Microb Inform Exp, 3(1):2, 2013.

J. C. Flanagan, J. M. Lang, A. E. Darling, J. A. Eisen, and D. A.

Coil. Draft Genome Sequence of Curtobacterium flaccumfaciens Strain 


\begin{tabular}{|c|c|}
\hline 1493 & UCD-AKU (Phylum Actinobacteria). Genome Announcements, \\
\hline 1494 & 1(3):e00244-13-e00244-13, May 2013. \\
\hline 1495 & P. Green. Phrap. version 1, page 090518., 2009. \\
\hline 1496 & W. P. Hanage, C. Fraser, and B. G. Spratt. Sequences, sequence \\
\hline 1497 & clusters and bacterial species. Philosophical Transactions of the Royal Society \\
\hline 1498 & B: Biological Sciences, 361(1475):1917-1927, Nov 2006. \\
\hline 1499 & H. E. Holland-Moritz, D. R. Bevans, J. M. Lang, A. E. Darling, \\
\hline 1500 & J. A. Eisen, and D. A. Coil. Draft Genome Sequence of Leucobacter sp. Strain \\
\hline 1501 & UCD-THU (Phylum Actinobacteria). Genome Announcements, \\
\hline 1502 & 1(3):e00325-13-e00325-13, Jun 2013. \\
\hline 1503 & D. H. Huson and C. Scornavacca. Dendroscope 3: An Interactive \\
\hline 1504 & Tool for Rooted Phylogenetic Trees and Networks. Systematic Biology, \\
\hline 1505 & 61(6):1061-1067, jul 2012. \\
\hline 1506 & A. O. Kislyuk, L. S. Katz, S. Agrawal, M. S. Hagen, A. B. Conley, \\
\hline 1507 & P. Jayaraman, V. Nelakuditi, J. C. Humphrey, S. A. Sammons, D. Govil, and \\
\hline 1508 & et al. A computational genomics pipeline for prokaryotic sequencing projects. \\
\hline 1509 & Bioinformatics, 26(15):1819-1826, Aug 2010. \\
\hline 1510 & David Coil; Guillaume Jospin; Jenna Lang;. Miscellaneous Scripts \\
\hline 1511 & for Workflow. \\
\hline 1512 & http://dx.doi.org/10.6084/m9.figshare.1086285, 2014. \\
\hline 1513 & J. R. Lo, J. M. Lang, A. E. Darling, J. A. Eisen, and D. A. Coil. \\
\hline 1514 & Draft Genome Sequence of an Actinobacterium, Brachybacterium muris Strain \\
\hline 1515 & UCD-AY4. Genome Announcements, 1(2):e00086-13-e00086-13, Mar 2013. \\
\hline
\end{tabular}


1516

1517

1518

1519

1520

1521

1522

1523

1524

1525

1526

1527

1528

1529

1530

1531

1532

1533

1534

1535

1536
JR Lo, JM Lang, AE Darling, JA Eisen, and DA Coil. Draft genome sequence of an Actinobacterium, Brachybacterium muris strain UCD-AY4. Genome Announc, 1:e0008613, Mar 2013.

T. Magoc, S. Pabinger, S. Canzar, X. Liu, Q. Su, D. Puiu, L. J. Tallon, and S. L. Salzberg. GAGE-B: an evaluation of genome assemblers for bacterial organisms. Bioinformatics, 29(14):1718-1725, Jul 2013.

[34] V. M. Markowitz, I.-M. A. Chen, K. Palaniappan, K. Chu, E. Szeto, M. Pillay, A. Ratner, J. Huang, T. Woyke, M. Huntemann, and et al. IMG 4 version of the integrated microbial genomes comparative analysis system. Nucleic Acids Research, 42(D1):D560-D567, Jan 2014.

[35] R. Overbeek, R. Olson, G. D. Pusch, G. J. Olsen, J. J. Davis, T. Disz, R. A. Edwards, S. Gerdes, B. Parrello, M. Shukla, and et al. The SEED and the Rapid Annotation of microbial genomes using Subsystems Technology (RAST). Nucleic Acids Research, 42(D1):D206-D214, Jan 2014.

[36] M. N. Price, P. S. Dehal, and A. P. Arkin. FastTree: Computing Large Minimum Evolution Trees with Profiles instead of a Distance Matrix. Molecular Biology and Evolution, 26(7):1641-1650, apr 2009.

S. L. Salzberg, A. M. Phillippy, A. Zimin, D. Puiu, T. Magoc, S. Koren, T. J. Treangen, M. C. Schatz, A. L. Delcher, M. Roberts, and et al. GAGE: A critical evaluation of genome assemblies and assembly algorithms. Genome Research, 22(3):557-567, Mar 2012. 
1537

1538

1539

1540

1541

1542

1543

1544

1545

1546

1547

1548

1549

1550

1551

1552

1553

1554

1555

1556

1557

1558
[38] F. Sanger, S. Nicklen, and A. R. Coulson. DNA sequencing with chain-terminating inhibitors. Proceedings of the National Academy of Sciences, 74(12):5463-5467, dec 1977.

[39] T. Seemann. Prokka: rapid prokaryotic genome annotation.

Bioinformatics, Mar 2014.

[40] J. T. Simpson and R. Durbin. Efficient construction of an assembly string graph using the FM-index. Bioinformatics, 26(12):i367-i373, Jun 2010.

[41] E. Stackebrandt. Report of the ad hoc committee for the re-evaluation of the species definition in bacteriology. INTERNATIONAL JOURNAL OF SYSTEMATIC AND EVOLUTIONARY MICROBIOLOGY, 52(3):1043-1047, May 2002.

B. J. Stucky. SeqTrace: A Graphical Tool for Rapidly Processing DNA Sequencing Chromatograms. Journal of Biomolecular Techniques, 23:90-93, 2012.

Andrew Tritt, Jonathan A. Eisen, Marc T. Facciotti, and Aaron E. Darling. An Integrated Pipeline for de Novo Assembly of Microbial Genomes. PLoS ONE, 7(9):e42304, Sep 2012. D. R. Zerbino and E. Birney. Velvet: Algorithms for de novo short read assembly using de Bruijn graphs. Genome Research, 18(5):821-829, Feb 2008. 


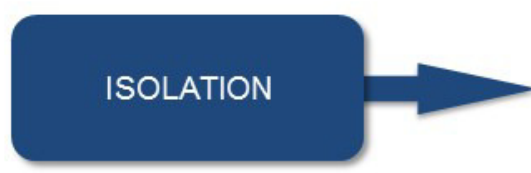

-Collection/plating

-Serial streaking to isolate
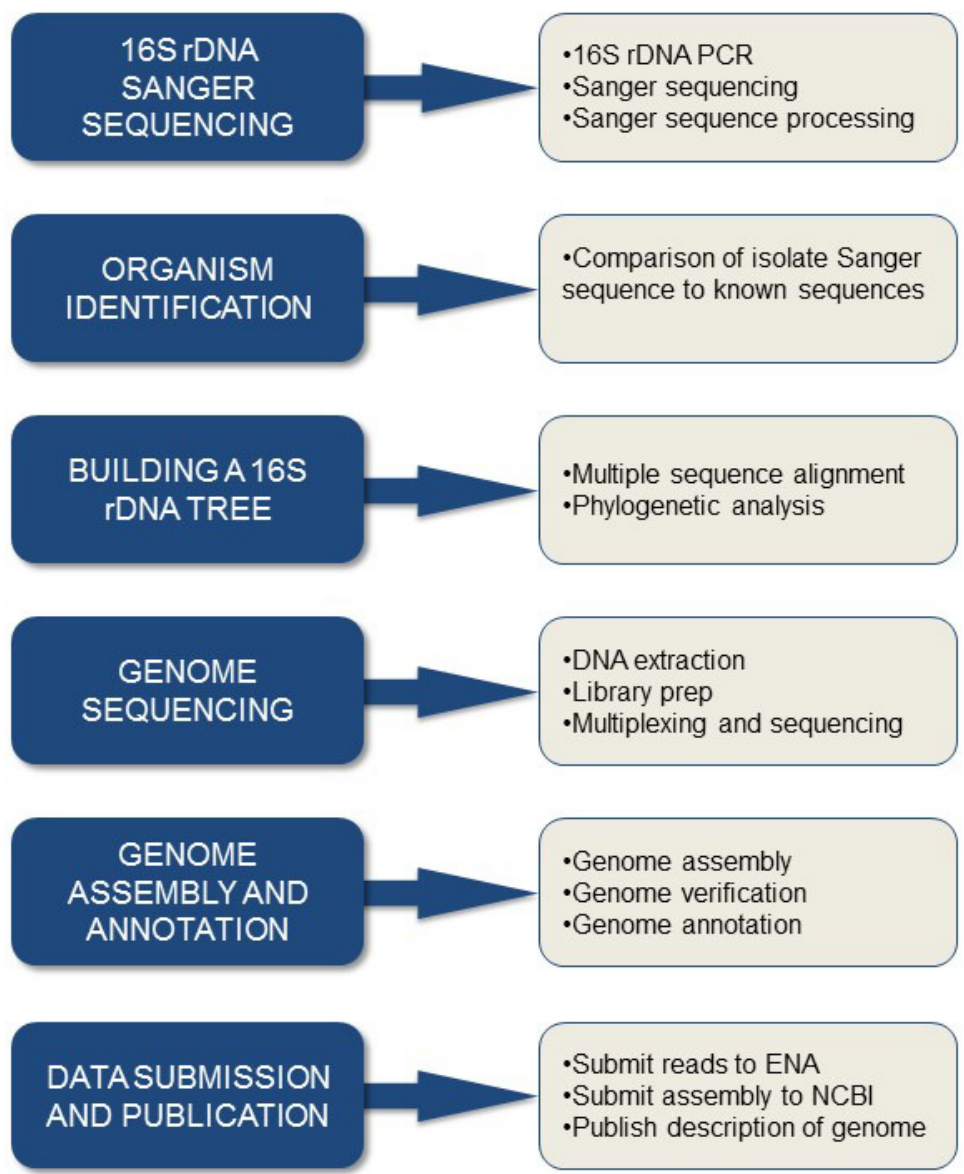

1559

1560 Figure 1: Overview of the Workflow

1561 All the steps required to go from a swab to a genome.

1562 


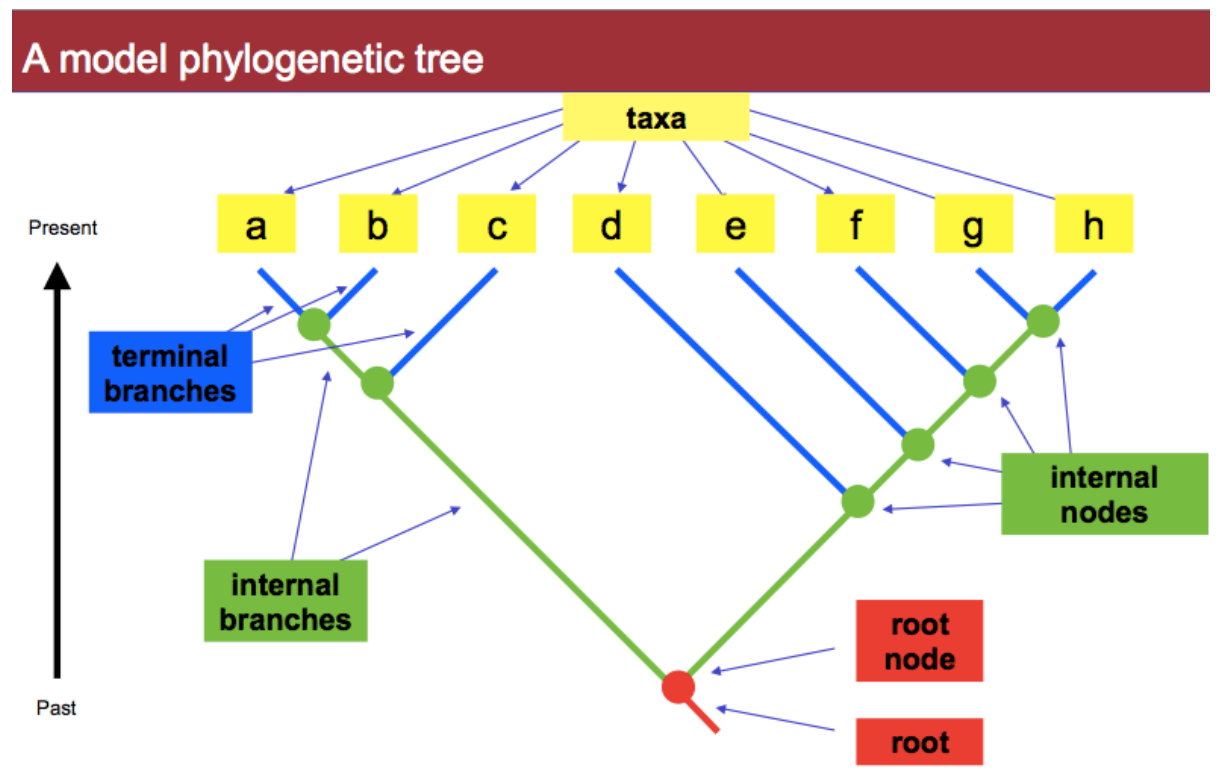

1563

1564 Figure 2: A model phylogenetic tree

1565 A phylogenetic tree is often helpful in assigning taxonomy to an

1566 unknown sequence.

1567

1568 
1569

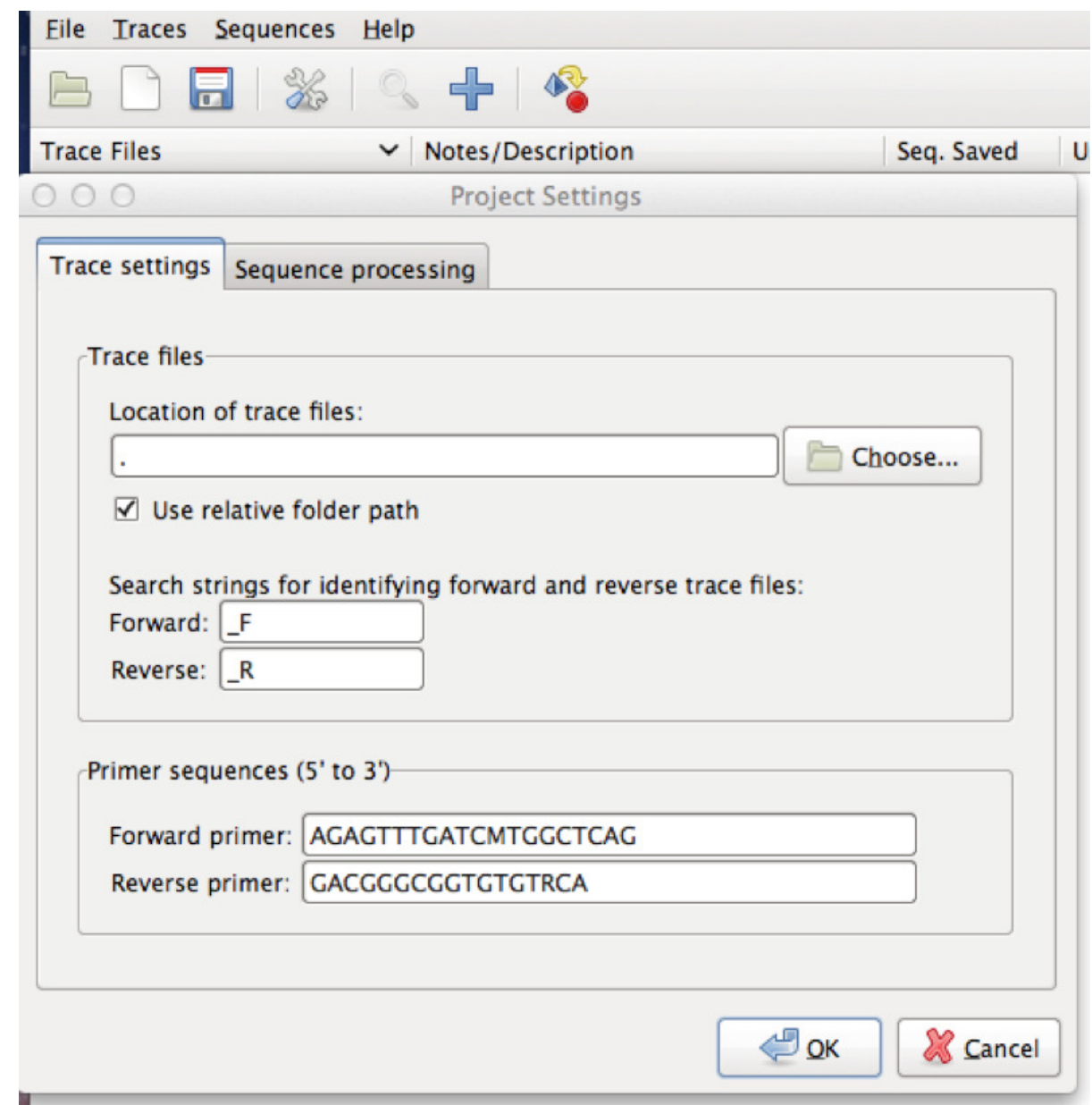

\section{$1570 \quad$ Figure 3: SeqTrace options}

1571 This screenshot shows an example of manually entered primer

1572 information in SeqTrace.

1573

1574 


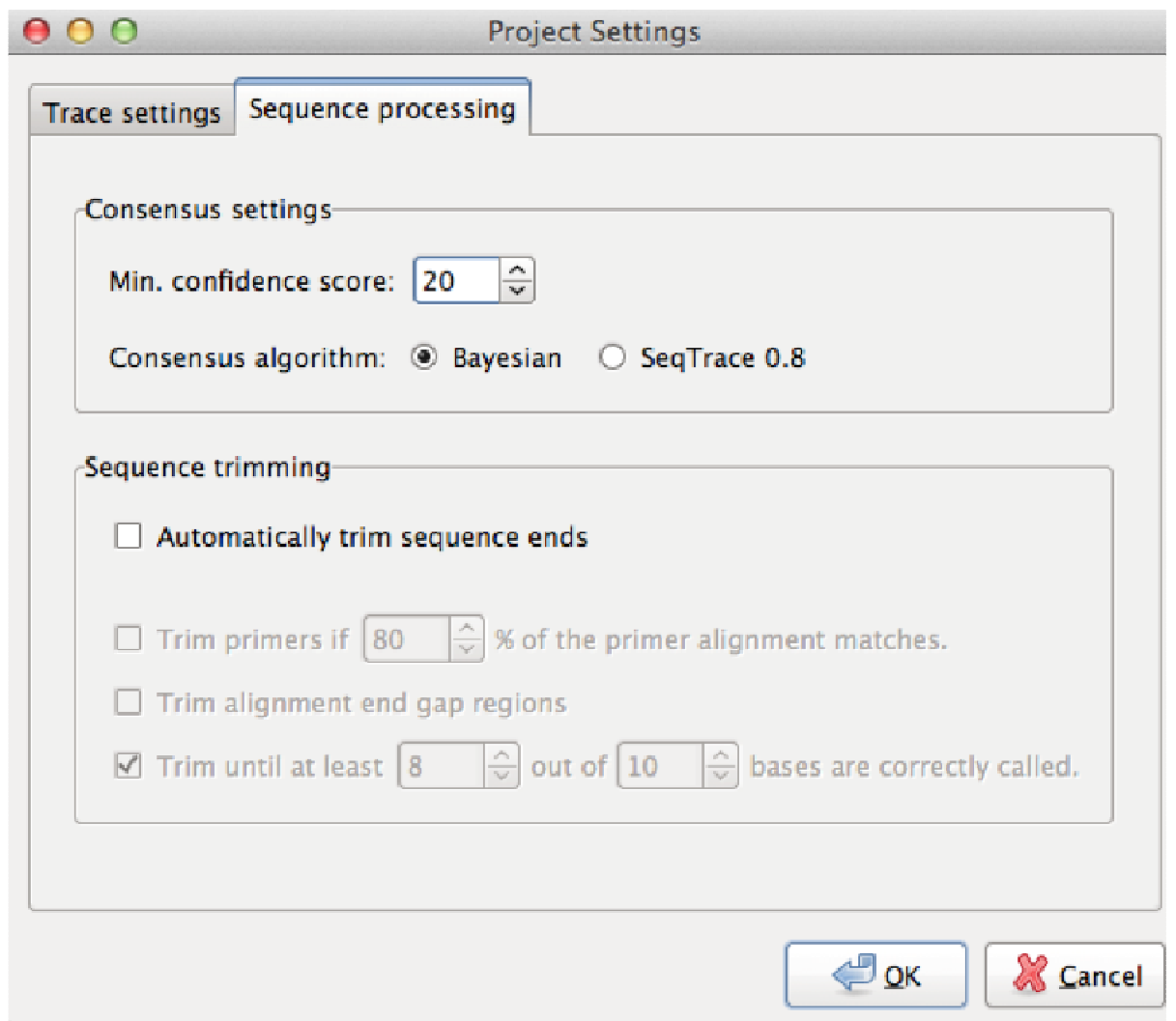

$1576 \quad$ Figure 4: SeqTrace trimming setting

1577 An example of reducing the minimum confidence score in SeqTrace.

1578

1579

1580

1581 


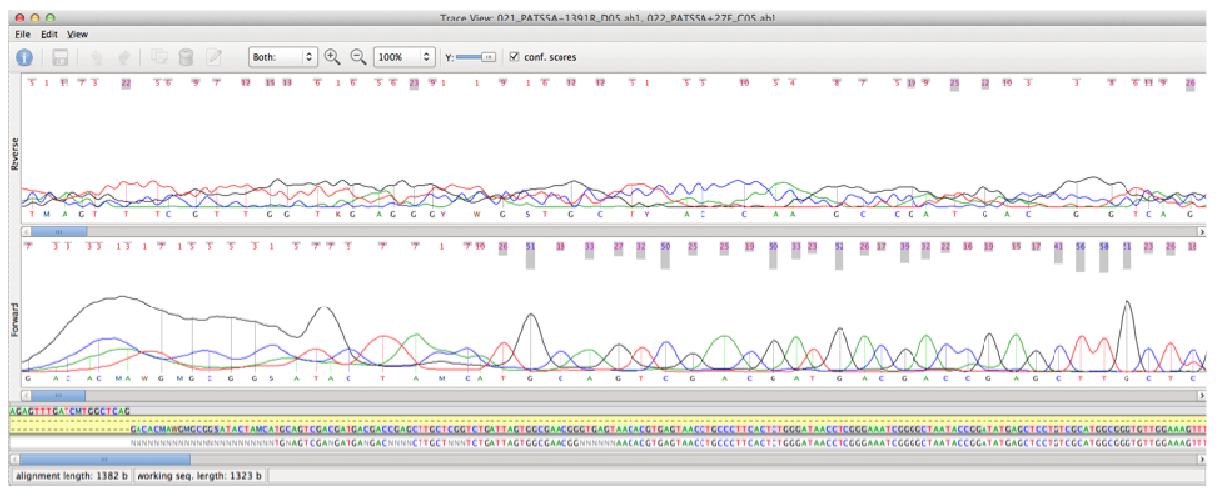

\section{$1583 \quad$ Figure 5: Sanger chromatogram}

1584 This screenshot from SeqTrace shows both the chromatogram (trace) as 1585 well as the consensus sequence.

1586

1587

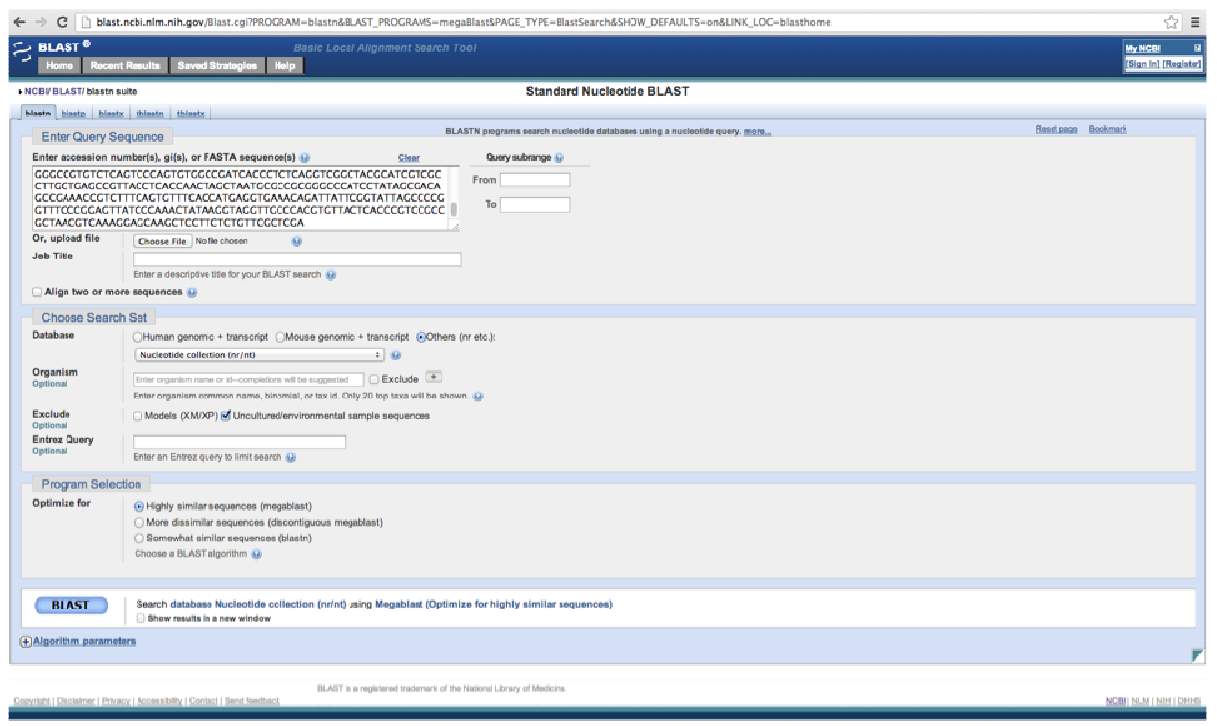

1589 Figure 6: BLAST options

1590 The recommended settings for using BLAST in this workflow.

1591 


\begin{tabular}{|c|c|c|}
\hline \multicolumn{3}{|l|}{ Quick Search } \\
\hline Search Field & & Search Term \\
\hline NCBI BioProject ID & $=\hat{\forall}$ & \\
\hline \multicolumn{3}{|l|}{ NCBI BioProject Accession } \\
\hline \multicolumn{3}{|l|}{ NCBI Locus Tag } \\
\hline Project Status & & Select from below... \\
\hline ITS SPID & $=\hat{\nabla}$ & \\
\hline Biosample Name & & Brachybacterium \\
\hline NCBI BioProject ID & $=\hat{\nabla}$ & \\
\hline Sequencing Project Search & Biosample Search & Analysis Project Search \\
\hline
\end{tabular}

1592

\section{$1593 \quad$ Figure 7: GOLD Search}

1594 Sample "Quick Search" page on GOLD.

1595

1596

1597 


\begin{tabular}{|c|c|c|}
\hline $\begin{array}{c}\text { GOLD Project ID } \\
+\end{array}$ & $\begin{array}{c}\text { Project Name } \\
+\end{array}$ & $\begin{array}{c}\text { Project Status } \\
+\end{array}$ \\
\hline Gp0001925 & Brachybacterium faecium 6-10, DSM 4810 & Complete and Published \\
\hline Gp0004437 & Brachybacterium muris & incomplete \\
\hline Gp0011776 & Brachybacterium paraconglomeratum LC44 & Permanent Draft \\
\hline Gp0012502 & Brachybacterium squillarum M-6-3 & Permanent Draft \\
\hline Gp0028874 & Brachybacterium alimentarium CNRZ 925 & incomplete \\
\hline Gp0028876 & Brachybacterium nesterenkovii CNRZ 926 & incomplete \\
\hline Gp0033260 & Brachybacterium muris UCD-AY4 & Permanent Draft \\
\hline$\underline{\text { Gp}} \underline{0035956}$ & Brachybacterium tyrofermentans CNRZ 926 & incomplete \\
\hline Gp0086679 & Brachybacterium phenoliresistens W13A50 & incomplete \\
\hline Gp0089909 & Brachybacterium phenoliresistens W13A50 & Permanent Draft \\
\hline Gp0093608 & Brachybacterium zhongshanense JCM 15471 & incomplete \\
\hline
\end{tabular}

RESET [1 - 11] of 11 Show $25 \div$ results.

1598

\section{$1599 \quad$ Figure 8: GOLD Results}

1600 Sample results for Brachybacterium on GOLD.

1601

1602 


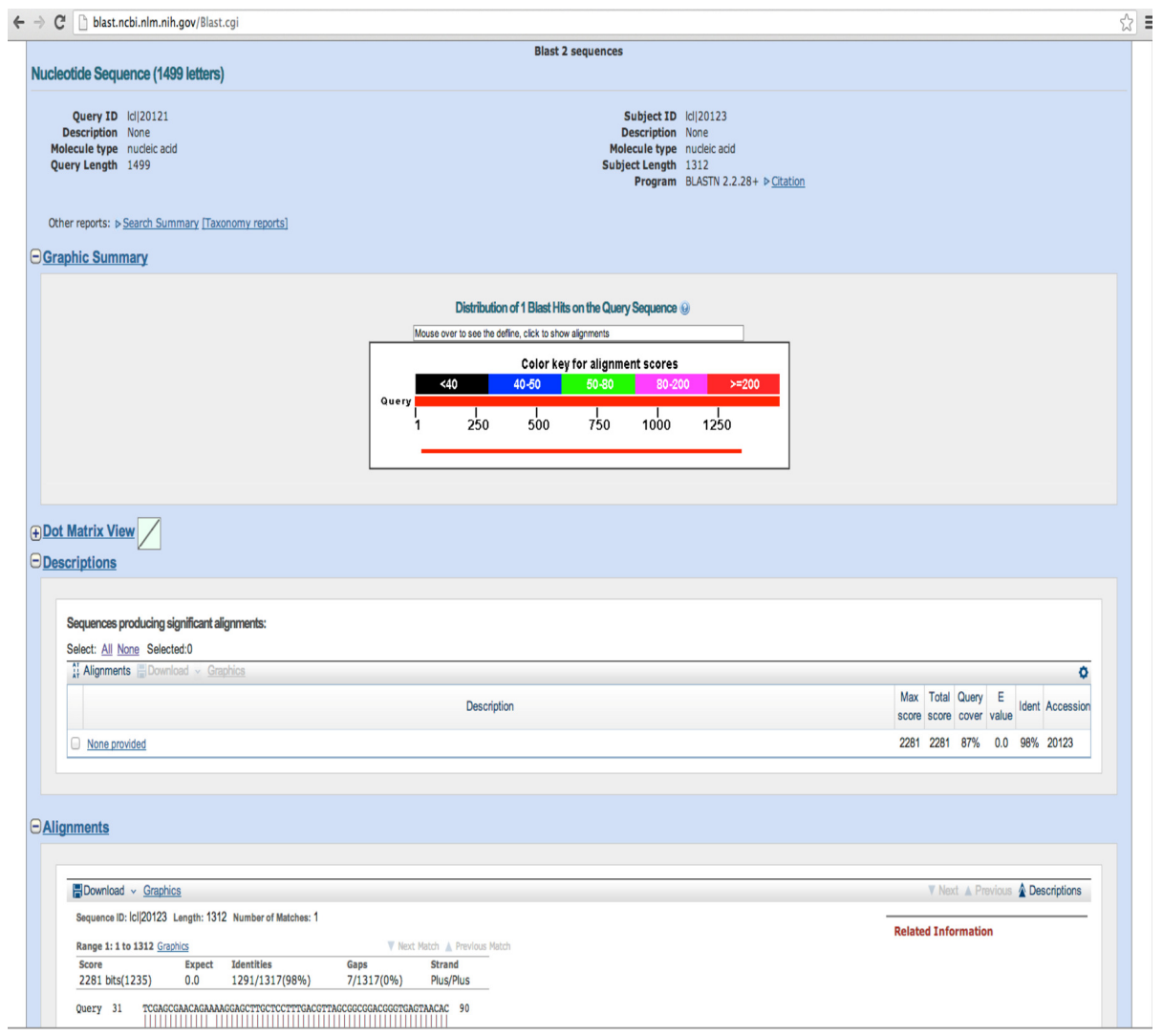

1604 Figure 9: Sample "Align Sequences Nucleotide BLAST" results

1605 In this example, our sequence of interest is $98 \%$ identical to the target 1606 sequence.

1607

1608

1609 


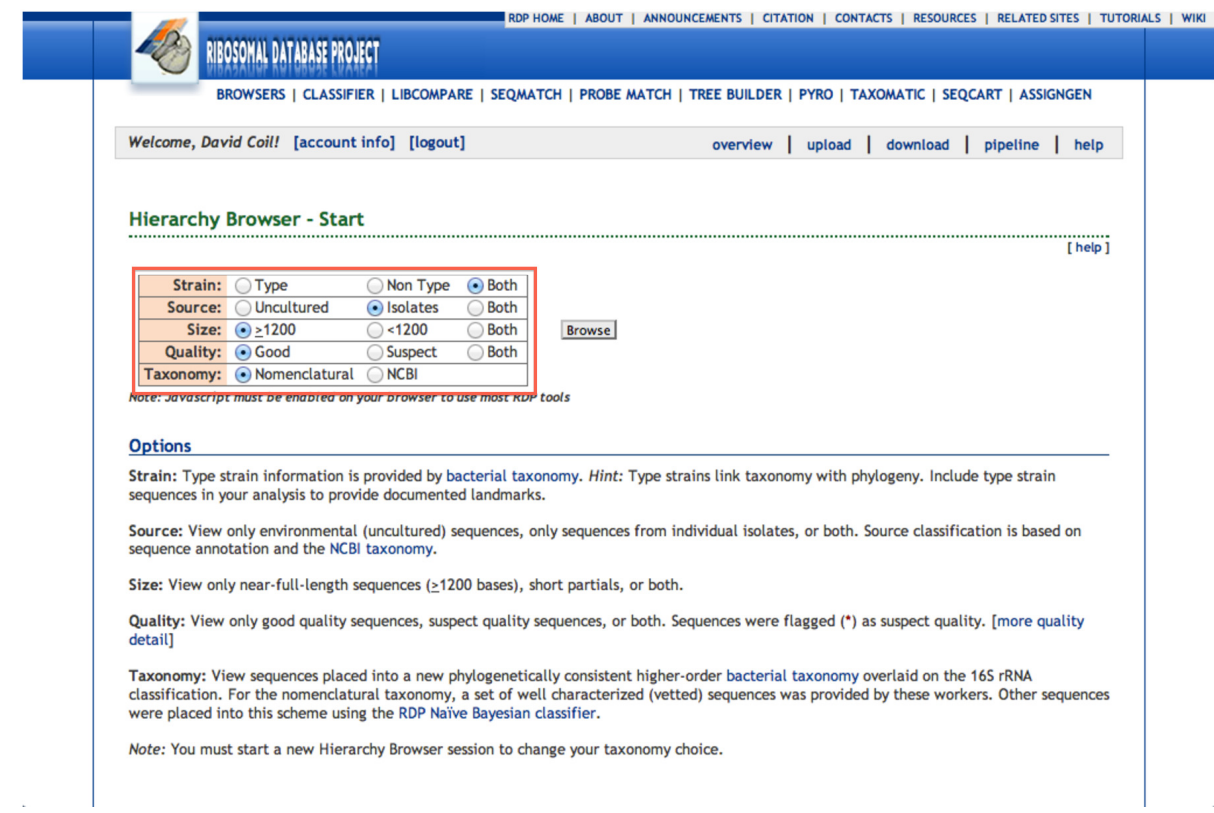

1611

1612 Figure 10: RDP Options

1613 Here we show our recommended options for RDP.

1614

1615

1616 


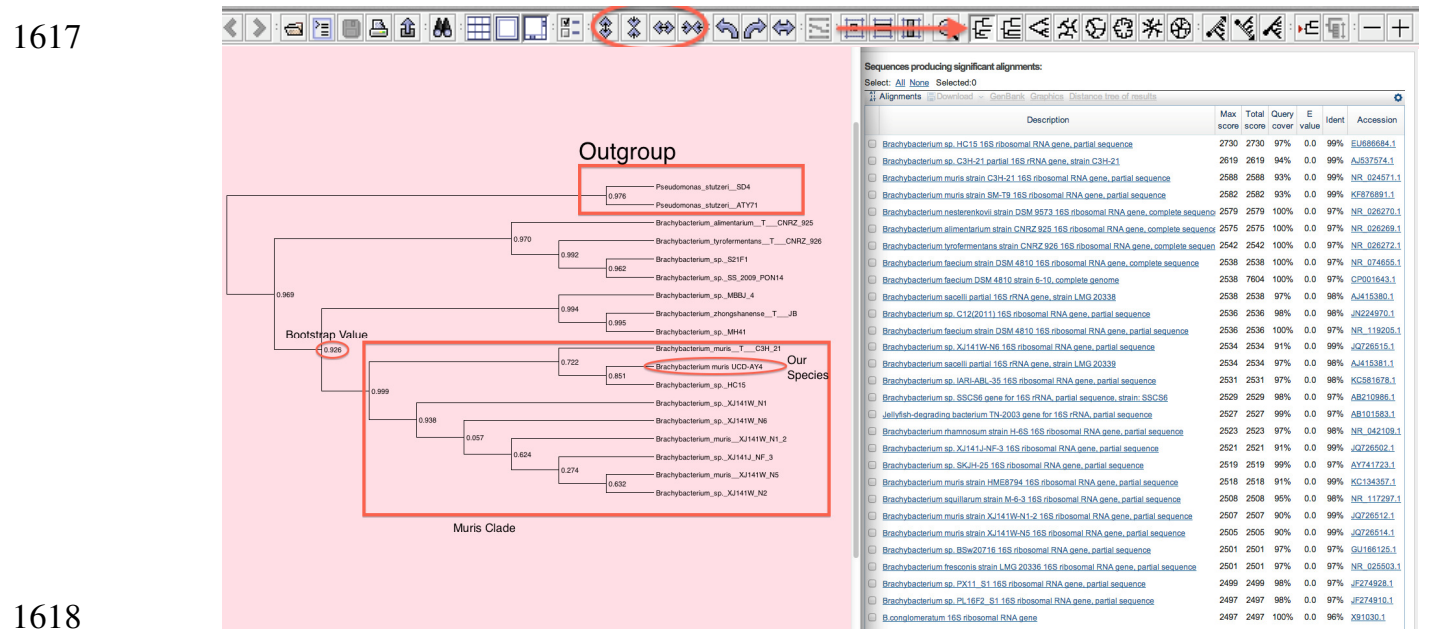

1619 Figure 12: An informative phylogenetic tree

1620 This phylogenetic tree shows our sequence of interest to be in a clade

1621 where everything has the same name.

1622
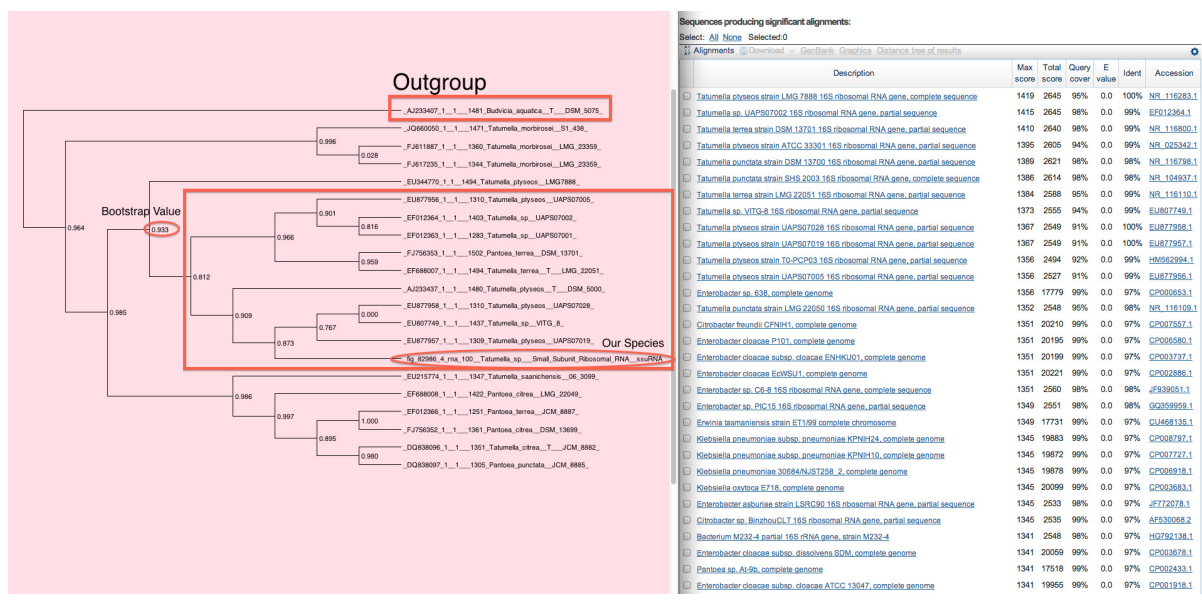

1624

1626 In this phylogenetic tree our species of interest is found in a clade with

1627 several species, some of which are found in other clades.

1628 


\begin{tabular}{|c|c|c|}
\hline Item & Best Case (Per Sample) & Worst Case (Per Sample) \\
\hline DNA Extraction ${ }^{1}$ & $\$ 1.66$ & $\$ 166$ \\
\hline $\mathrm{PCR}^{2}$ & $\$ 0.60$ & $\$ 150$ \\
\hline PCR Cleanup ${ }^{3}$ & $\$ 2.00$ & $\$ 100$ \\
\hline Sanger $^{4}$ & $\$ 14.00$ & $\$ 14$ \\
\hline Library Prep ${ }^{5}$ & $\$ 58.33$ & $\$ 2,800$ \\
\hline Illumina Sequencing ${ }^{6}$ & $\$ 35.42$ & $\$ 1,700$ \\
\hline Total & $\$ 112.01$ & $\$ 4,930$ \\
\hline
\end{tabular}

1630

1631

1632

1633

1634

1635

1636

1637 
1638 Estimated Materials Costs of Bacterial Genome Sequencing

1639 Table 1-This table shows the estimated materials (i.e. without labor)

1640 cost of performing a genome sequencing project with this workflow in

1641 2014. The "Best Case" shows the marginal cost of sequencing one

1642 genome in a case where you are multiplexing 48 samples, and have the

1643 appropriate kits and reagents on hand. The "Worst Case" shows the

1644 cost of doing a single genome, with no multiplexing, in a lab where

1645 every reagent needed to be purchased new and was not used for

1646 anything else. Specific assumptions are as follows; ${ }^{1}$ This assumes the

1647 purchase of a standard DNA extraction kit, good for 100 samples. ${ }^{2}$ This

1648 assumes purchase of a standard 200U PCR reagent kit. ${ }^{3}$ PCR cleanup

1649 can be performed in a number of ways; gel extraction, beads, or

1650 columns for example. Here we assume purchase of a standard

1651 column-based kit. ${ }^{4}$ Sanger sequencing cost is given as the price per

1652 reaction (\$7 at our sequencing facility), times the forward and reverse

1653 reactions. ${ }^{5}$ This assumes the purchase of a 48 -sample Nextera or

1654 TrueSeq kit from Illumina, however kits from other manufacturers can

1655 be cheaper. ${ }^{6}$ Our sequencing cost estimate assumes purchase of an

1656 Illumina MiSeq run from a sequencing facility.

1657

1658 